Review

\title{
The Influence of the Process Parameters on the Mechanical Properties of PLA Specimens Produced by Fused Filament Fabrication-A Review
}

\author{
Vasile Cojocaru $^{1}$ D, Doina Frunzaverde ${ }^{1, * \mathbb{D}}$, Calin-Octavian Miclosina ${ }^{1}$ and Gabriela Marginean ${ }^{2}$ \\ 1 Department of Engineering Science, Babeș-Bolyai University, P-ța Traian Vuia, Nr. 1-4, \\ 320085 Resita, Romania; vasile.cojocaru@ubbcluj.ro (V.C.); calin.miclosina@ubbcluj.ro (C.-O.M.) \\ 2 Department of Materials Science and Testing, Westphalian University of Applied Sciences Gelsenkirchen \\ Bocholt Recklinghausen, Neidenburgerstr. 43, 45897 Gelsenkirchen, Germany; gabriela.marginean@w-hs.de \\ * Correspondence: doina.frunzaverde@ubbcluj.ro
}

check for

updates

Citation: Cojocaru, V.; Frunzaverde,

D.; Miclosina, C.-O.; Marginean, G.

The Influence of the Process

Parameters on the Mechanical

Properties of PLA Specimens

Produced by Fused Filament

Fabrication-A Review. Polymers

2022, 14, 886. https://doi.org/

$10.3390 /$ polym 14050886

Academic Editor: Petr Smolka

Received: 31 January 2022

Accepted: 21 February 2022

Published: 23 February 2022

Publisher's Note: MDPI stays neutral with regard to jurisdictional claims in published maps and institutional affiliations.

Copyright: (C) 2022 by the authors. Licensee MDPI, Basel, Switzerland. This article is an open access article distributed under the terms and conditions of the Creative Commons Attribution (CC BY) license (https:// creativecommons.org/licenses/by/ $4.0 /)$.

\begin{abstract}
Polylactic acid (PLA) is produced from renewable materials, has a low melting temperature and has a low carbon footprint. These advantages have led to the extensive use of polylactic acid in additive manufacturing, particularly by fused filament fabrication (FFF). PLA parts that are 3D printed for industrial applications require stable mechanical properties and predictability regarding their dependence on the process parameters. Therefore, the development of the FFF process has been continuously accompanied by the development of software packages that generate CNC codes for the printers. A large number of user-controllable process parameters have been introduced in these software packages. In this respect, a lot of articles in the specialized literature address the issue of the influence of the process parameters on the mechanical properties of 3D-printed specimens. A systematic review of the research targeting the influence of process parameters on the mechanical properties of PLA specimens additively manufactured by fused filament fabrication was carried out by the authors of this paper. Six process parameters (layer thickness, printing speed, printing temperature, build plate temperature, build orientation and raster angle) were followed. The mechanical behavior was evaluated by tensile, compressive and bending properties.
\end{abstract}

Keywords: polylactic acid (PLA); fused filament fabrication (FFF); mechanical properties; process parameters

\section{Introduction}

Additive manufacturing (AM) technologies are increasingly used for component fabrication and tend to become an essential topic of the Industry 4.0 concept [1]. These technologies shorten the manufacturing time, thereby allowing the rapid transition from 3D models to real parts. Using additive manufacturing, both the external and the internal geometry of components can be optimized. The optimization of the internal geometry of parts allows for an efficient material distribution, correlated to the stress state.

The ISO/ASTM 52900:2015 standard [2] defines the following categories of processes used for additive manufacturing of polymers: material extrusion, material jetting, powder bed fusion, binder jetting, vat photo-polymerization and sheet lamination.

Fused filament fabrication (FFF) is a material extrusion process in which the part is built up by successive layers, each of them being made line by line. The material, in the form of a continuous filament, is melted and deposited by a printing head with a nozzle. Fused filament fabrication (also known as fused deposition modeling) is currently one of the most widely used additive technologies [1]. A great variety of equipment has been developed for the FFF technology, ranging from industrial and laboratory use to office and hobby applications.

The advantages of fused filament fabrication are based on the simplicity of the process and on the low cost of materials, equipment and consumables [3]. For the widespread 
use of fused filament fabrication for industrial manufacturing, it is necessary to obtain printed products with predictable properties. The following categories of AM product requirements are defined by ISO 17296-3:2014 [4]:

- $\quad$ Surface requirements: surface texture, appearance, color;

- Geometric requirements: linear and angular dimensions, dimensional tolerances, geometrical tolerances (deviations in shape and relative position);

- Mechanical requirements: hardness, tensile strength, impact strength, compressive strength, flexural strength, fatigue strength, creep, ageing, frictional coefficient, shear resistance and crack extension;

- $\quad$ Build material requirements: density, physical properties and chemical properties.

The mechanical properties of components obtained by fused filament fabrication are influenced not only by the material properties, but also by the characteristics of the 3D printer, the process parameters and the post-process treatments [5-9].

The 3D model conversion for the printing process is achieved by using a slicer software (a G-code generation software, specific to the printing process). This software allows for setting the values for a large number of process parameters, the most frequently analyzed being the following [6]:

- $\quad$ Slicing parameters: layer thickness, printing speed/flow rate, nozzle diameter, raster parameters, number of wall lines, wall thickness, top layer thickness, bottom layer thickness;

- $\quad$ Temperature parameters: printing head temperature, build plate temperature, build volume temperature (printer with/without closed space), cooling;

- Infill parameters: infill density and infill pattern;

- $\quad$ Build orientation parameters and the use of support material.

Polylactic acid (PLA) is a thermoplastic polyester that can be obtained from renewable resources at a low production cost. PLA has a low melting point, making it easy to use in most FFF equipment. The extrusion temperature of PLA is lower than that of other common polymeric materials (ABS-acrylonitrile butadiene styrene, PEEK-polyether ether ketone, PETG-polyethylene terephthalateglycol), and its tensile strength and elastic modulus may be superior to ABS and PET-G [10-13]. Furthermore, PLA is biodegradable, has a low carbon footprint and low smoke emissions during extrusion [13] and can be successfully used in medical applications, because it is not metabolically harmful [14].

The influence of the process parameters on the mechanical properties of PLA specimens obtained by fused filament fabrication has been intensively studied in recent years. In the research carried out so far, one to five process parameters have been varied. Statistical methods, such as design of experiments (DOE), the Taguchi method, and analysis of variance (ANOVA) were used to determine the influence of the different parameters on the mechanical characteristics $[15,16]$.

In order to understand the effect of each of these numerous parameters, as well as the correlation between them, a systematic analysis of the published research is necessary. Therefore, the goal of this paper is to present an up-to-date review of the literature targeting the influence of the process parameters on the mechanical properties of PLA specimens, made by fused filament fabrication. The analysis focused on the variation of the following parameters: layer thickness, printing speed, printing head temperature, build plate temperature, build orientation and raster angle. For the characterization of the static mechanical behavior, the results of tensile, bending and compression tests were followed. The literature review was performed following the Preferred Reporting Items for Systematic Reviews and Meta-Analyses (PRISMA) guidelines.

The search terms used in bibliographic databases to select the analyzed papers were as follows: (PLA OR *poly\$lactic*) AND (FDM OR FFF OR *fused*) AND (mechanical OR tensile OR bending OR strength). Papers dealing with the dynamic behavior of PLA, with PLA-based composites or only with the variation of mechanical properties as a function of the infill pattern and the infill density were not included in the present review. As 
the variation of mechanical properties according to the type of infill has been intensively studied, an analysis of the influence of the infill pattern and the infill density on the mechanical behavior of printed parts will be presented by the authors in a separate paper.

Table 1 shows the notations and abbreviations used in this paper to define the process parameters and mechanical characteristics.

Table 1. Notations and abbreviations.

\begin{tabular}{|c|c|c|}
\hline Process Parameter & Notation & Units \\
\hline Layer thickness (layer height) & $\mathrm{t}$ & $(\mathrm{mm})$ \\
\hline Printing speed & $\mathrm{s}_{\mathrm{p}}$ & $(\mathrm{mm} / \mathrm{s})$ \\
\hline Printing head (nozzle) temperature & $\mathrm{T}_{\mathrm{H}}$ & $\left({ }^{\circ} \mathrm{C}\right)$ \\
\hline Build plate temperature & $\mathrm{T}_{\mathrm{B}}$ & $\left({ }^{\circ} \mathrm{C}\right)$ \\
\hline Nozzle diameter & $d_{n}$ & $(\mathrm{~mm})$ \\
\hline Filament diameter & $d_{f}$ & $(\mathrm{~mm})$ \\
\hline $\begin{array}{l}\text { Build orientation (acc. to ISO/ASTM 52921:2013 [17]) } \\
\text { first letter-axis parallel to the longest dimension of part; } \\
\text { second letter-axis parallel to second longest dimension } \\
\text { of part }\end{array}$ & $\begin{array}{l}X Y, X Z \\
Y X, Y Z \\
Z X, Z Y\end{array}$ & $(-)$ \\
\hline $\begin{array}{c}\text { Build orientation angle in the xy plane (around the } z \text {-axis) } \\
\text { Indexes represent the reference build orientation from } \\
\text { which angle is measured ( } \alpha_{Z X}=0^{\circ} \text { correspond to } Z X \\
\text { build orientation); }\end{array}$ & $\alpha_{Y X}, \alpha_{X Y}, \alpha_{X Z}$ & $\left({ }^{\circ}\right)$ \\
\hline Build orientation angle in the yz plane (around the $\mathrm{x}$-axis) & $\beta_{X Y}, \beta_{Y X}, \beta_{X Z}$ & $\left({ }^{\circ}\right)$ \\
\hline Build orientation angle in the $\mathrm{xz}$ plane (around the $\mathrm{y}$-axis) & $\gamma_{Z X}, \gamma_{X Y}, \gamma_{X Z}$ & $\left({ }^{\circ}\right)$ \\
\hline Raster angle & $\theta$ & $\left({ }^{\circ}\right)$ \\
\hline Number of wall lines & $\mathrm{W}_{\mathrm{L}}$ & $(-)$ \\
\hline Tensile/bending test speed & $\mathrm{S}_{\mathrm{t}}$ & $(\mathrm{mm} / \mathrm{min})$ \\
\hline Ultimate tensile strength & UTS & $(\mathrm{MPa})$ \\
\hline Ultimate flexural strength & UFS & $(\mathrm{MPa})$ \\
\hline Modulus of elasticity (Young's modulus) & $\mathrm{E}$ & (MPa) \\
\hline
\end{tabular}

\section{From Pre-Process Conditions to Mechanical Characterization of FFF PLA}

The systematic analysis of trends in the variation of the mechanical properties of PLA components as a function of one or more process parameters must take into consideration the values used for all factors that may influence these characteristics. Therefore, the operational conditions for all the steps involved, from filament production to mechanical testing, should be known.

In this connection, at least the following defining phases of the production and testing processes should be taken into account: (a) the manufacturing and storage conditions of the filament; (b) the design of the product and the selection of the infill parameters; (c) the selection of the process parameters; $(\mathrm{d})$ type of the 3D printing equipment; (e) post-process treatments, storage conditions and ageing; and (f) the mechanical tests conditions.

\subsection{The Manufacturing and Storage Conditions of the Filament}

In fused filament fabrication processes, filaments with a circular cross-section and a diameter of $1.75 \mathrm{~mm}$ or $2.85 \mathrm{~mm}$ are used. Up to $17 \%$ variation of the ultimate tensile strength of specimens was pointed out in [18], using PLA filaments from different manufacturers. Significant differences (approximately $18 \%$ of the ultimate flexural strength) were also obtained by the bending test [19]. These differences may occur due to the manufacturing conditions or to the filament storage conditions. For example, the humidity of the filament storage enclosure can cause changes in the printing behavior and thereby noticeable variations of the mechanical properties. The color of the filament can also influence the mechanical characteristics of PLA specimens [20,21]. 


\subsection{The Design of the Model and the Selection of the Infill Parameters}

In the design process, the shape and dimensions of the part are determined. For 3D-printing fabrication, the output of the design must be a 3D model of the part, exported as a stl file. The quality of the stl file can influence the dimensional accuracy of the part. As the surfaces of CAD models are converted into meshes of triangles in stl files, an increase in the number of triangles leads to better quality, but also to an increase in stl file size.

The designer has also to define the infill settings that will be used. The selection of the infill pattern and density must be correlated with the stress and strain states for the future product. Both parameters are major factors of influence on the mechanical properties of 3D FFF-printed components [22-25]. The infill characteristics are to be set into the slicer software (addressed in the next step), but it is important to highlight that the infill selection is part of the design process of the component.

\subsection{The Selection of the Process Parameters}

This step involves the positioning of the part onto the printer's build space, the choice of the values for the slicing parameters, and the setting of the temperature conditions. The G-code generated in the slicer software controls the printing process.

The selection of the process parameters must be correlated with the anisotropic behavior of PLA components manufactured by fused filament fabrication. Build orientation and raster settings have a major influence on the anisotropy of printed parts [26-29].

The designer must also consider at this stage the possibility of making the part with or without the use of support material. The use of support material can influence the surface quality and mechanical behavior of the products.

The high influence of the number of specimens printed simultaneously on the flexural strength of rectangular hollow cross-section specimens is highlighted in [30].

\subsection{The Type of the 3D-Printing Equipment}

The printing equipment can influence the dimensional accuracy and mechanical behavior of fused filament fabricated parts. Vettori and co-authors [8] present a roundrobin study performed on PLA printed on different FFF equipment, using the same process parameters. The results show the important differences of the ultimate tensile strength values $(\mathrm{max}=54.2 \mathrm{MPa}$, $\min =13.2 \mathrm{MPa}$ ) depending on the printer used.

Temperature variations may occur in printing on open workspace equipment. Different mechanical properties can be achieved in these situations for identical components placed in different areas of the workspace. The use of closed space equipment with heat flow control can lead to optimized temperature distribution [31].

\subsection{The Post-Process Treatments, Storage and Ageing}

The mechanical behavior of FFF 3D-printed components can be influenced by postprinting thermal or thermo-chemical treatments, as well as by material ageing [32]. In [33] is highlighted the improvement of thermomechanical properties of PLA specimens subjected to post-print annealing. However, in [34] it is shown that PLA specimens obtained by FFF and annealed at $60-120^{\circ} \mathrm{C}$ for $30-60$ min showed a decrease in the modulus of elasticity and the ultimate tensile strength.

The properties of components made of PLA can be modified by the storage environmental conditions and the storage duration. Moreover, the dimensional accuracy of printed PLA components can be influenced by material volume changes and residual stress occurrence caused by the PLA crystallinity [35].

At low humidity, PLA has higher mechanical strength but lower toughness [36]. In [37] it is shown that reducing moisture content from $10 \%$ to $1 \%$ results in a decrease in the tensile strength with $24.4 \%$.

In [38] are presented the variations of yield strength and modulus of elasticity as functions of the ageing time for printed PLA. An improvement of the mechanical characteristics 
is observed with an increase in the ageing duration. Contrariwise, ageing for $240 \mathrm{~h}$ in a salt-fog environment causes a decrease of about $20 \%$ of the tensile strength [39].

\subsection{The Mechanical Tests Conditions}

The most commonly used testing methods for the characterization of the mechanical behavior of fused filament fabricated PLA parts are tensile tests and three-point bending tests [40]. At the moment, there are no specific ISO or ASTM standards defining the shape of specimens manufactured by FFF additive manufacturing. Thus, for the tensile tests "dog-bone" specimens defined by the general standards for plastics are used: ASTM D63814 [41] and ISO 527-2:2012 [42]. The specimens used for the bending tests are defined by: ASTM D790-10 [43] and ISO 178:2019 [44].

The use of specimens with different shapes and dimensions may result in different mechanical characteristics. In [28], the authors estimate that the UTS values obtained by tests made on ASTM D638-Type IV specimens may be overestimated compared to the values resulting from tests performed on ASTM D638-Type I specimens.

One of the main problems highlighted in several papers refers to the occurrence of the breakage outside the gauge length of the tensile specimens. This improper failure may be related to the geometry of the dog-bone specimen, which leads to stress concentrations in the radius area [1]. Sierra et al. [45] studied the tensile behavior of ASTM D638 specimens with modified radius and conclude that the radius influences the mechanical strength obtained in tests. In [1] it is shown that an alternative to specimens with radius is the use of prismatic specimens defined by ISO 527-5 [46] and ASTM D3039 [47].

Valean et al. show that increasing the thickness of the specimen decreases the values of the mechanical characteristics UTS and E determined by tensile tests [48].

The variation of the mechanical properties of PLA specimens depending on the tensile test speed and the tensile test strain rate was analyzed in $[49,50]$. Vidakis and coauthors conclude that the tensile strength of PLA is strongly influenced by the strain rate and tensile test speed. The increase in the test speed from $10 \mathrm{~mm} / \mathrm{min}$ to $100 \mathrm{~mm} / \mathrm{min}$ leads to an increase in the tensile strength values by approximately $11 \%$ [50].

\section{Layer Thickness}

The layer thickness (or layer height) is the height of each deposited layer (Figure 1). For the top and the bottom layer, respectively, a distinct thickness can be set. It should be noted that the layer thickness is correlated with the diameter of the nozzle and the width of the raster.

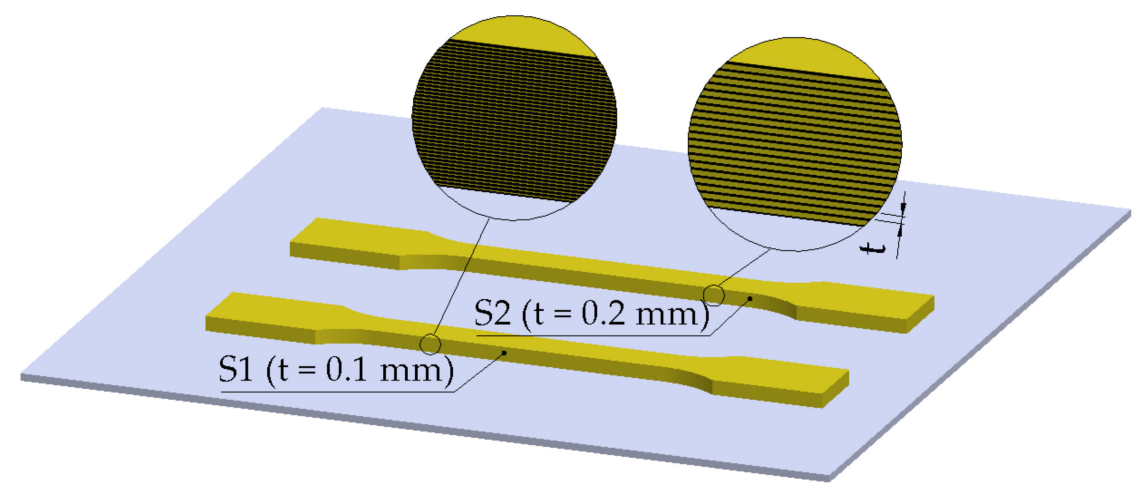

Figure 1. Layer thickness (t) for ISO 527-2 Type 1A tensile test specimens (S1, S2).

In the research analyzed in this paper, the layer thickness was varied in the range of $0.06-0.6 \mathrm{~mm}$, with the most commonly analyzed values situated between $0.10 \mathrm{~mm}$ and $0.30 \mathrm{~mm}$. Selecting higher values for layer thickness leads to shorter production times, but also to lower part resolution. On the other hand, working with lower layer thicknesses determines longer durations of the printing processes and higher part resolution. The total number of layers is the ratio of the part height on the z-axis to the layer thickness (reference 
system for upward building, according to ISO/ASTM 52921:2013). A 50\% decrease in the layer thickness results in a doubling of the printing time. Increasing the number of layers emphasizes the re-heat effect for deposited layers, leading to improved diffusion and adhesion between layers.

It should be noted that the variation of the mechanical properties with the layer thickness is influenced also by other parameters (Table 2). For example, in [51] it is shown that the dependence of the tensile strength on the layer thickness is affected by the raster type. On the other hand, the influence of the nozzle diameter of the printing head is greater than the influence of the layer thickness when a high yield strength is desired for a product [52]. Triyono et al. [53] indicate that the increase in the nozzle diameter leads to an increase in the density and the tensile strength of 3D-printed products. The authors consider that these two interconnected effects can be attributed to better interfacial bonding between the in-plane raster lines. At the same ratio between layer thickness and nozzle diameter, the adhesion between adjacent lines improves with the increases in the nozzle diameter. For big nozzle diameters, the raster lines were discovered to be even slightly overlapped.

Table 2. The influence of the layer thickness on the mechanical properties of FFF-printed PLA.

\begin{tabular}{|c|c|c|c|c|c|c|c|c|c|}
\hline \multirow[b]{2}{*}{ Ref. } & \multicolumn{7}{|c|}{ FFF Process Parameters } & \multirow{2}{*}{$\begin{array}{l}\text { Mechanical } \\
\text { Test Settings }\end{array}$} & \multirow{2}{*}{$\begin{array}{l}\text { Results and } \\
\text { Conclusions }\end{array}$} \\
\hline & $t(\mathrm{~mm})$ & $\begin{array}{c}\mathrm{s}_{\mathrm{p}} \\
(\mathrm{mm} / \mathrm{s})\end{array}$ & $\begin{array}{c}\mathrm{T}_{\mathrm{H}} \\
\left({ }^{\circ} \mathrm{C}\right)\end{array}$ & $\begin{array}{c}\mathrm{T}_{\mathrm{B}} \\
\left({ }^{\circ} \mathrm{C}\right)\end{array}$ & $\begin{array}{c}\text { B.O. } \\
(-)\end{array}$ & $\begin{array}{ll}\theta \\
\left({ }^{\circ}\right)\end{array}$ & $\begin{array}{c}\text { Other } \\
\text { Parameters }\end{array}$ & & \\
\hline \multicolumn{10}{|c|}{$\begin{array}{l}\mathrm{t} \text {-layer thickness (layer height); } \mathrm{s}_{\mathrm{p}}-\text { printing speed; } \mathrm{T}_{\mathrm{H}}-\text { printing head (nozzle) temperature; } \mathrm{T}_{\mathrm{B}} \text { - build plate temperature; } \\
\text { B.O.-build orientation; } \theta-\text { raster angle; } \mathrm{d}_{\mathrm{f}}-\text { filament diameter; } \mathrm{d}_{\mathrm{n}} \text {-nozzle diameter; } \mathrm{W}_{\mathrm{L}}-\text { number of wall lines. }\end{array}$} \\
\hline [19] & $0.06-0.60$ & 25 & - & - & Vertical & - & $\begin{array}{c}\mathrm{d}_{\mathrm{f}}=2.85 \mathrm{~mm} ; \\
\mathrm{d}_{\mathrm{n}}=0.4-0.8 \mathrm{~mm}\end{array}$ & $\begin{array}{c}\text { Bending, } \\
\text { rectangular } \\
\text { hollow } \\
\text { cross-section; } \\
\mathrm{s}_{\mathrm{t}}=10 \mathrm{~mm} / \mathrm{min}\end{array}$ & $\begin{array}{c}\text { UFS increases with the } \\
\text { increase in the } d_{n} / t \text { ratio. } \\
\text { UFS for } t=0.06, \\
d_{n}=0.40 \text { about } 3.9 \times \\
\text { higher than UFS for } \\
t=0.4, d_{n}=0.40 .\end{array}$ \\
\hline [23] & $0.10-0.20$ & $20-40$ & 210 & - & $X Y$ & $45^{\circ} /-45^{\circ}$ & $\begin{array}{l}\mathrm{d}_{\mathrm{f}}=1.75 \mathrm{~mm} ; \\
\mathrm{d}_{\mathrm{n}}=0.4 \mathrm{~mm} ; \\
20-80 \% \text { infill }\end{array}$ & $\begin{array}{c}\text { Tensile_ASTM } \\
\text { D638 }\end{array}$ & $\begin{array}{l}\text { Low increase in UTS } \\
\text { with the decrease in } \\
\text { layer thickness. }\end{array}$ \\
\hline [24] & $0.10-0.30$ & 30 & 195 & 110 & Horizontal & $40^{\circ}-80^{\circ}$ & $\begin{array}{l}\mathrm{d}_{\mathrm{f}}=1.75 \mathrm{~mm} \\
\mathrm{~d}_{\mathrm{n}}=0.3 \mathrm{~mm} \\
20-80 \% \text { infill }\end{array}$ & $\begin{array}{c}\text { Tensile-ASTM } \\
\text { D638 }\end{array}$ & $\begin{array}{l}\text { The variation of UTS vs. } \\
\text { layer thickness is } \\
\text { influenced by the } \\
\text { raster angle. }\end{array}$ \\
\hline [26] & $0.06-0.24$ & $20-80$ & 210 & - & $\begin{array}{l}\text { YX; YZ; } \\
\text { ZY }\end{array}$ & $0^{\circ}$ & $\begin{array}{c}\mathrm{d}_{\mathrm{f}}=1.75 \mathrm{~mm} \\
\mathrm{~d}_{\mathrm{n}}=0.4 \mathrm{~mm} \\
100 \% \text { infill }\end{array}$ & $\begin{array}{l}\text { Tensile-ASTM } \\
\text { D638; Bending- } \\
\text { ASTM } \\
\text { D790 }\end{array}$ & $\begin{array}{c}\text { Highest UTS }(89.1 \mathrm{MPa}) \\
\text { for } \mathrm{t}=0.06, \\
\mathrm{~s}_{\mathrm{p}}=50 \mathrm{~mm} / \mathrm{s}, \mathrm{YX} \\
\text { specimens. } \\
\text { Highest UFS }(65 \mathrm{MPa}) \\
\text { for } \mathrm{t}=0.06, \\
\mathrm{~s}_{\mathrm{p}}=80 \mathrm{~mm} / \mathrm{s}, \\
\mathrm{YZ} \text { specimens. }\end{array}$ \\
\hline [28] & $0.06-0.50$ & $30-200$ & $175-230$ & - & $X Y ; Z X$ & - & $\begin{array}{c}\mathrm{d}_{\mathrm{f}}=1.75 \mathrm{~mm} ; \\
\mathrm{d}_{\mathrm{n}}=0.5 \mathrm{~mm} ; \\
100 \% \text { infill }\end{array}$ & $\begin{array}{c}\text { Tensile-ASTM } \\
\text { D638, Type I vs. } \\
\text { Type IV }\end{array}$ & $\begin{array}{l}\text { UTS decreases with the } \\
\text { increase in the } \\
\text { layer thickness. }\end{array}$ \\
\hline [32] & $0.10-0.30$ & 60 & 215 & 60 & Horizontal & $\begin{array}{l}45^{\circ} /-45^{\circ} ; \\
0^{\circ} / 90^{\circ} ; \\
0^{\circ} /-30^{\circ} / \\
30^{\circ} /-60^{\circ} / \\
60^{\circ} / 90^{\circ} /\end{array}$ & $\begin{array}{c}\mathrm{d}_{\mathrm{f}}=1.75 \mathrm{~mm} ; \\
100 \% \text { infill; } \\
\text { ageing; } \\
\text { heat treatment }\end{array}$ & $\begin{array}{l}\text { Tensile-ASTM } \\
\text { D638 }\end{array}$ & $\begin{array}{c}\text { Higher UTS for } \\
\text { specimens with } \\
\mathrm{t}=0.1 \mathrm{~mm} \text {. The decrease } \\
\text { in UTS for } \mathrm{t}=0.3 \mathrm{~mm} \text { vs. } \\
\mathrm{t}=0.1 \mathrm{~mm} \text { is higher for } \\
\text { aged specimens, with } \\
\text { and without } \\
\text { heat treatment. }\end{array}$ \\
\hline [51] & $0.06-0.35$ & 60 & $190-220$ & 60 & $X Y$ & $\begin{array}{r}0^{\circ} ; 90^{\circ} ; \\
45^{\circ} /-45^{\circ}\end{array}$ & $\begin{array}{c}\mathrm{d}_{\mathrm{f}}=1.75 \mathrm{~mm} ; \\
\mathrm{d}_{\mathrm{n}}=0.4 \mathrm{~mm} ; \\
100 \% \text { infill; } \\
\mathrm{W}_{\mathrm{L}}=2\end{array}$ & $\begin{array}{l}\text { Tensile-ASTM } \\
\text { D638, Type I } \\
\text { specimens; } \\
\mathrm{s}_{\mathrm{t}}=5 \mathrm{~mm} / \mathrm{min}\end{array}$ & $\begin{array}{l}\text { The variation of UTS } \\
\text { with layer thickness is } \\
\text { influenced by } \theta \text {. For } \\
\theta=0^{\circ} \text { the highest UTS is } \\
\text { obtained for } \mathrm{t}=0.06 \mathrm{~mm} \text {. } \\
\text { High variation of UTS } \\
\text { vs. } \mathrm{t} \text { for } \theta=90^{\circ} \text {. }\end{array}$ \\
\hline [54] & $0.10-0.40$ & 90 & 185 & - & $\mathrm{Z}$ & - & $\begin{array}{c}\mathrm{d}_{\mathrm{f}}=1.75 \mathrm{~mm} \\
100 \% \text { infill }\end{array}$ & $\begin{array}{l}\text { Tensile-ASTM } \\
\text { D638 IV; } \\
\mathrm{s}_{\mathrm{t}}=5 \mathrm{~mm} / \mathrm{min} \\
\end{array}$ & $\begin{array}{l}\text { Highest UTS and E for } \\
\qquad t=0.4 \mathrm{~mm} \text {. }\end{array}$ \\
\hline [55] & $0.20-0.40$ & 50 & $190-210$ & - & Horizontal & - & $\begin{array}{c}\mathrm{d}_{\mathrm{f}}=2.85 \mathrm{~mm} ; \\
20-100 \% \text { infill; } \\
\mathrm{W}_{\mathrm{L}}=2\end{array}$ & $\begin{array}{l}\text { Tensile-ASTM } \\
\text { D638; increased } \\
\text { specimen } \\
\text { thickness; } \\
\mathrm{s}_{\mathrm{t}}=5 \mathrm{~mm} / \mathrm{min}\end{array}$ & $\begin{array}{c}\text { Highest UTS } \\
\text { (61.66 MPa) and } \mathrm{E} \\
(3815.50 \mathrm{MPa}) \text { for } \\
\mathrm{t}=0.3 \mathrm{~mm} .\end{array}$ \\
\hline
\end{tabular}


Table 2. Cont.

\begin{tabular}{|c|c|c|c|c|c|c|c|c|c|}
\hline \multirow[b]{2}{*}{ Ref. } & \multicolumn{7}{|c|}{ FFF Process Parameters } & \multirow{2}{*}{$\begin{array}{l}\text { Mechanical } \\
\text { Test Settings }\end{array}$} & \multirow{2}{*}{$\begin{array}{l}\text { Results and } \\
\text { Conclusions }\end{array}$} \\
\hline & $\mathrm{t}(\mathrm{mm})$ & $\begin{array}{c}\mathrm{s}_{\mathrm{p}} \\
(\mathrm{mm} / \mathrm{s})\end{array}$ & $\begin{array}{c}\mathrm{T}_{\mathrm{H}} \\
\left({ }^{\circ} \mathrm{C}\right)\end{array}$ & $\begin{array}{c}\mathrm{T}_{\mathrm{B}} \\
\left({ }^{\circ} \mathrm{C}\right)\end{array}$ & $\underset{(-)}{\text { B.O. }}$ & $\begin{array}{c}\theta \\
\left({ }^{\circ}\right)\end{array}$ & $\begin{array}{c}\text { Other } \\
\text { Parameters }\end{array}$ & & \\
\hline [56] & $0.10-0.40$ & 50-150 & 190-205 & - & Horizontal & - & $\begin{array}{c}\mathrm{d}_{\mathrm{f}}=1.75 \mathrm{~mm} \\
\mathrm{~d}_{\mathrm{n}}=0.4 \mathrm{~mm} \\
\text { cooling fan }\end{array}$ & $\begin{aligned} & \text { Tensile; } \\
& \mathrm{s}_{\mathrm{t}}=5 \mathrm{~mm} / \mathrm{min}\end{aligned}$ & $\begin{array}{l}\text { Highest UTS } \\
(60.26 \mathrm{MPa}) \text { for } \\
\mathrm{t}=0.10 \mathrm{~mm} \text {; layer } \\
\text { thickness was the } \\
\text { dominant factor } \\
\text { for UTS. }\end{array}$ \\
\hline [57] & $0.10-0.30$ & 50 & 210 & 60 & $\begin{array}{l}\alpha_{X Y}= \\
0^{\circ}-60^{\circ}\end{array}$ & - & $\begin{array}{c}\mathrm{d}_{\mathrm{f}}=1.75 \mathrm{~mm} ; \\
\mathrm{d}_{\mathrm{n}}=0.4 \mathrm{~mm} ; \\
20-80 \% \text { infill; } \\
\mathrm{W}_{\mathrm{L}}=2\end{array}$ & $\begin{array}{l}\text { Tensile-ASTM } \\
\text { D638; } \\
\text { Bending- } \\
\text { ASTM D790; } \\
\mathrm{s}_{\mathrm{t}}=1 \mathrm{~mm} / \mathrm{min}\end{array}$ & $\begin{array}{c}\text { Highest UTS obtained } \\
\text { for } \mathrm{t}=0.2 \mathrm{~mm} \text { and } \\
\alpha_{\mathrm{XY}}=30^{\circ} \text { at } 80 \% \text { infill } \\
\text { density; } \\
\text { Highest UFS obtained } \\
\text { for } \mathrm{t}=0.3 \mathrm{~mm} \text { and } \\
\alpha_{X Y}=0^{\circ} \text { at } 80 \% \\
\text { infill density. }\end{array}$ \\
\hline [58] & $0.125-0.25$ & - & - & - & $\begin{array}{l}\alpha_{X Y}=0^{\circ} ; \\
\alpha_{X Y}=45^{\circ}\end{array}$ & - & $50-90 \%$ infill & $\begin{array}{c}\text { Tensile-ISO } \\
527 \\
\end{array}$ & $\begin{array}{c}\text { Higher UTS for } \\
\mathrm{t}=0.25 \mathrm{~mm} .\end{array}$ \\
\hline [59] & $0.10-0.35$ & $40-80$ & 220 & 25 & $\begin{array}{l}\alpha_{X Y}= \\
0^{\circ}-90^{\circ}\end{array}$ & - & $\begin{array}{c}\mathrm{d}_{\mathrm{f}}=1.75 \mathrm{~mm} \\
100 \% \text { infill }\end{array}$ & $\begin{array}{c}\text { Tensile-ASTM } \\
\text { D638, Type V } \\
\text { specimens } \\
\end{array}$ & $\begin{array}{l}\text { Higher E and UTS for } \\
\text { low values of the } \\
\text { layer thickness. }\end{array}$ \\
\hline$[60]$ & $0.05-0.40$ & 60 & 200 & - & $\begin{array}{l}\text { Horizontal; } \\
\text { Vertical }\end{array}$ & - & $\begin{array}{c}\mathrm{d}_{\mathrm{f}}=1.75 \mathrm{~mm} ; \\
60 \% \text { infill; } \\
\text { variable cooling }\end{array}$ & Tensile & $\begin{array}{c}\text { Highest UTS } \\
(53.62 \mathrm{MPa}) \text { at } \\
\mathrm{t}=0.2 \mathrm{~mm} \text {, for } \\
\text { horizontal } \\
\text { printed specimens. }\end{array}$ \\
\hline$[61]$ & $0.20-0.30$ & $38-52$ & 190 & 40 & - & $0^{\circ} ; 90^{\circ}$ & $\begin{array}{c}\mathrm{d}_{\mathrm{n}}=0.40 \mathrm{~mm} \\
40 \% \text { infill }\end{array}$ & $\begin{array}{c}\text { Bending- } \\
\text { ASTM D790; } \\
\mathrm{s}_{\mathrm{t}}=12 \mathrm{~mm} / \mathrm{min}\end{array}$ & $\begin{array}{l}\text { Higher flexural strength } \\
\text { for } \mathrm{t}=0.2 \mathrm{~mm} \text {. }\end{array}$ \\
\hline$[62]$ & $0.10-0.30$ & $25-75$ & 210 & 60 & Vertical & - & $\begin{array}{c}\mathrm{d}_{\mathrm{n}}=0.40 \mathrm{~mm} ; \\
\quad \text { four FFF } \\
\text { printers (P1-P4) }\end{array}$ & $\begin{array}{l}\text { Bending, } \\
\text { rectangular } \\
\text { hollow } \\
\text { cross-section; } \\
\mathrm{s}_{\mathrm{t}}=10 \mathrm{~mm} / \mathrm{min}\end{array}$ & $\begin{array}{l}\text { P1-P2: UFS and sample } \\
\text { mass decrease with } \\
\text { thickness. } \\
\text { P3-P4: maximum UFS } \\
\text { for } \mathrm{t}=0.15 \mathrm{~mm} \text { and } \\
\mathrm{t}=0.20 \mathrm{~mm} .\end{array}$ \\
\hline [63] & $0.10-0.20$ & 60 & 205 & 60 & Horizontal & $\begin{array}{c}0^{\circ} ; 18^{\circ} ; 45^{\circ} \\
72^{\circ} ; 90^{\circ}\end{array}$ & $\begin{array}{l}100 \text { infill; } \\
W_{L}=2-6\end{array}$ & $\begin{array}{l}\text { Tensile-ASTM } \\
\text { D638 modified } \\
\text { specimens }\end{array}$ & $\begin{array}{c}\text { Low variation of UTS } \\
\text { and E with } \mathrm{t} \text {. Highest } \\
\text { UTS (49.29 MPa) and E } \\
(3497 \mathrm{MPa}) \text { for } \\
\mathrm{t}=0.10 \mathrm{~mm} .\end{array}$ \\
\hline$[64]$ & $0.10-0.30$ & - & 210 & 80 & $\begin{array}{l}\gamma \times Y= \\
0^{\circ}-90^{\circ}\end{array}$ & $\begin{array}{l}30^{\circ} ; 45^{\circ} \\
60^{\circ}\end{array}$ & $\begin{array}{c}\mathrm{d}_{\mathrm{f}}=1.75 \mathrm{~mm} \\
50 \% \text { infill }\end{array}$ & $\begin{array}{c}\text { Tensile_ASTM } \\
\text { D638 }\end{array}$ & $\begin{array}{l}\text { UTS decreases with the } \\
\text { increase in the } \\
\text { layer thickness. }\end{array}$ \\
\hline [65] & $0.10-0.30$ & $30-90$ & $210-230$ & $50-80$ & $X Y$ & $0^{\circ} / 90^{\circ}$ & $\begin{array}{c}\mathrm{d}_{\mathrm{f}}=1.75 \mathrm{~mm} ; \\
\mathrm{d}_{\mathrm{n}}=0.4 \mathrm{~mm} ; \\
100 \% \text { infill; } \\
\mathrm{W}_{\mathrm{L}}=2\end{array}$ & $\begin{array}{c}\text { Tensile-ISO } \\
527-2 ; \\
\mathrm{s}_{\mathrm{t}}=50 \mathrm{~mm} / \mathrm{min}\end{array}$ & $\begin{array}{l}\text { Higher UTS for } \\
t=0.2 \mathrm{~mm}\end{array}$ \\
\hline [66] & $0.10-0.20$ & $40-80$ & 220 & 60 & $X Y ; X Z$ & - & $\begin{array}{c}\mathrm{d}_{\mathrm{n}}=0.4 \mathrm{~mm} \\
100 \% \text { infill; } \\
\mathrm{W}_{\mathrm{L}}=3\end{array}$ & $\begin{array}{c}\text { Tensile-_ISO } \\
527 ; \\
\mathrm{s}_{\mathrm{t}}=5 \mathrm{~mm} / \mathrm{min}\end{array}$ & $\begin{array}{c}\text { Higher UTS }(46.22 \mathrm{MPa}) \\
\text { for XZ specimens with } \\
\mathrm{t}=0.1 \mathrm{~mm}, \\
\mathrm{~s}_{\mathrm{p}}=80 \mathrm{~mm} / \mathrm{s} .\end{array}$ \\
\hline [67] & $0.10-0.20$ & 60 & 200 & 60 & Horizontal & - & $\begin{array}{c}\mathrm{d}_{\mathrm{f}}=1.75 \mathrm{~mm} \\
\mathrm{~d}_{\mathrm{n}}=0.4 \mathrm{~mm} \\
50-100 \% \text { infill }\end{array}$ & $\begin{array}{c}\text { Tensile-ISO } \\
527-2\end{array}$ & $\begin{array}{l}\text { Low variation of UTS } \\
\text { and E with the layer } \\
\text { thickness. Higher UTS } \\
\text { for } \mathrm{t}=0.1 \mathrm{~mm} \text {. }\end{array}$ \\
\hline [68] & $0.10-0.40$ & 60 & 230 & 80 & Horizontal & - & $\begin{array}{c}\mathrm{d}_{\mathrm{f}}=1.75 \mathrm{~mm} \\
100 \% \text { infill }\end{array}$ & $\begin{array}{c}\text { Tensile-ASTM } \\
\text { D638; } \\
\text { Bending- } \\
\text { ASTM D790; } \\
\text { Impact-ISO } \\
180\end{array}$ & $\begin{array}{l}\text { UTS, UFS and Izod } \\
\text { impact strength decrease } \\
\text { with the increase in } \\
\text { layer thickness for all } \\
\text { raster patterns. }\end{array}$ \\
\hline [69-71] & $0.10-0.30$ & 50 & 210 & 70 & - & $\begin{array}{c}0^{\circ} ; 45^{\circ} \\
90^{\circ}\end{array}$ & $\begin{array}{c}\mathrm{d}_{\mathrm{f}}=1.75 \mathrm{~mm} ; \\
\mathrm{d}_{\mathrm{n}}=0.4 \mathrm{~mm} \\
100 \% \text { infill; } \\
\mathrm{W}_{\mathrm{L}}=1\end{array}$ & $\begin{array}{c}\text { Tensile-ASTM } \\
\text { D638; } \\
\text { Bending- } \\
\text { ASTM D790; } \\
\text { Impact_ASTM } \\
\text { D256 } \\
\end{array}$ & $\begin{array}{l}\text { UTS and UFS decrease } \\
\text { with the increase in the } \\
\text { layer thickness. Izod } \\
\text { impact strength } \\
\text { increases with the } \\
\text { layer thickness. }\end{array}$ \\
\hline [72] & $0.10-0.20$ & 30 & 200 & 50 & $\begin{array}{l}X Y ; X Z ; \\
Z X\end{array}$ & $45^{\circ} /-45^{\circ}$ & $\begin{array}{l}\mathrm{d}_{\mathrm{f}}=1.75 \mathrm{~mm} \\
\mathrm{~d}_{\mathrm{n}}=0.4 \mathrm{~mm} ; \\
20-50 \% \text { infill }\end{array}$ & $\begin{array}{c}\text { Tensile-ASTM } \\
\text { D638; } \\
\mathrm{s}_{\mathrm{t}}=5 \mathrm{~mm} / \mathrm{min}\end{array}$ & $\begin{array}{l}\text { Approx. } 10.6 \% \text { higher } \\
\text { UTS for } \mathrm{t}=0.10 \mathrm{~mm} \\
\text { compared to } \\
\mathrm{t}=0.20 \mathrm{~mm} .\end{array}$ \\
\hline [73] & $0.10-0.30$ & 20 & 210 & 50 & - & - & $\mathrm{d}_{\mathrm{f}}=1.75 \mathrm{~mm}$ & $\begin{array}{c}\text { Tensile, } \mathrm{s}_{\mathrm{t}}=1 \\
\mathrm{~mm} / \mathrm{min}\end{array}$ & $\begin{array}{c}\text { Higher UTS }(61.5 \mathrm{MPa}) \\
\text { for } \mathrm{t}=0.30 \mathrm{~mm} .\end{array}$ \\
\hline [74] & $0.05-0.20$ & 60 & 195-230 & 60 & $\begin{array}{l}\beta_{Y Z}= \\
0^{\circ}-90^{\circ}\end{array}$ & - & $\begin{array}{c}\mathrm{d}_{\mathrm{f}}=1.75 \mathrm{~mm} \\
\mathrm{~d}_{\mathrm{n}}=0.4 \mathrm{~mm}\end{array}$ & $\begin{array}{c}\text { Tensile--ISO } \\
527-2 ; \\
\mathrm{s}_{\mathrm{t}}=2 \mathrm{~mm} / \mathrm{min}\end{array}$ & $\begin{array}{l}\text { Low decrease in UTS } \\
\text { with the increase in the } \\
\text { layer thickness. }\end{array}$ \\
\hline
\end{tabular}


Table 2. Cont.

\begin{tabular}{|c|c|c|c|c|c|c|c|c|c|}
\hline \multirow[b]{2}{*}{ Ref. } & \multicolumn{7}{|c|}{ FFF Process Parameters } & \multirow{2}{*}{$\begin{array}{l}\text { Mechanical } \\
\text { Test Settings }\end{array}$} & \multirow{2}{*}{$\begin{array}{l}\text { Results and } \\
\text { Conclusions }\end{array}$} \\
\hline & $\mathrm{t}(\mathrm{mm})$ & $\begin{array}{c}\mathrm{s}_{\mathrm{p}} \\
(\mathrm{mm} / \mathrm{s})\end{array}$ & $\begin{array}{c}\mathrm{T}_{\mathrm{H}} \\
\left({ }^{\circ} \mathrm{C}\right)\end{array}$ & $\begin{array}{c}\mathrm{T}_{\mathrm{B}} \\
\left({ }^{\circ} \mathrm{C}\right)\end{array}$ & $\underset{(-)}{\text { B.O. }}$ & $\begin{array}{c}\theta \\
\left({ }^{\circ}\right)\end{array}$ & $\begin{array}{c}\text { Other } \\
\text { Parameters }\end{array}$ & & \\
\hline [75] & $0.10-0.20$ & 80 & 200 & 60 & $X Y$ & $45^{\circ}$ & $\begin{array}{c}\mathrm{d}_{\mathrm{f}}=1.75 \mathrm{~mm} ; \\
25-100 \% \text { infill; } \\
\text { variable } \\
\text { flow rate }\end{array}$ & $\begin{array}{l}\text { Tensile-ASTM } \\
\text { D638, Type IV }\end{array}$ & $\begin{array}{l}\text { Highest UTS } \\
(40.07 \mathrm{MPa}) \text { for } \\
\mathrm{t}=0.15 \mathrm{~mm}\end{array}$ \\
\hline$[76,77]$ & $0.10-0.30$ & 60 & 215 & - & $\begin{array}{l}\gamma \times z= \\
0^{\circ}-90^{\circ}\end{array}$ & - & $\mathrm{d}_{\mathrm{f}}=1.75 \mathrm{~mm}$ & $\begin{array}{c}\text { Tensile-ISO } \\
527-2 ; \\
\mathrm{s}_{\mathrm{t}}=0.1 \\
\mathrm{~mm} / \mathrm{min}\end{array}$ & $\begin{array}{c}\text { Highest UTS for } \\
t=0.10 \mathrm{~m} \text {. Low } \\
\text { variation of UTS and E } \\
\text { with layer thickness. }\end{array}$ \\
\hline [78] & $0.10-0.60$ & - & - & - & $\begin{array}{l}\gamma \times z= \\
0^{\circ}-90^{\circ}\end{array}$ & - & $\begin{array}{c}\mathrm{d}_{\mathrm{f}}=1.75 \mathrm{~mm} \\
\mathrm{~d}_{\mathrm{n}}=0.4 \mathrm{~mm}\end{array}$ & $\begin{array}{c}\text { Tensile-ISO } \\
527-2 ; \\
\mathrm{s}_{\mathrm{t}}=0.1 \\
\mathrm{~mm} / \mathrm{min}\end{array}$ & $\begin{array}{l}\text { Low variation of UTS } \\
\text { with layer thickness. }\end{array}$ \\
\hline [79] & $0.10-0.30$ & - & 220 & 60 & $\begin{array}{l}\gamma \times z= \\
0^{\circ}-90^{\circ}\end{array}$ & - & $\mathrm{d}_{\mathrm{f}}=1.75 \mathrm{~mm}$ & $\begin{array}{c}\text { Tensile-ISO } \\
527-2\end{array}$ & $\begin{array}{c}\text { Highest UTS for } \\
t=0.10 \mathrm{~mm} \text { and } \\
t=0.20 \mathrm{~mm} \text {. Low } \\
\text { variation of UTS vs. } t .\end{array}$ \\
\hline
\end{tabular}

\section{Printing Speed}

The printing speed $(\mathrm{mm} / \mathrm{s})$ is the speed of the printing head in the $X Y$ plane during the deposition of the layers. This parameter is correlated with the flow rate $\left(\mathrm{mm}^{3} / \mathrm{s}\right)$.

In the research analyzed in this paper (Table 3 ) the printing speed was varied in the range of $20 \mathrm{~mm} / \mathrm{s}-170 \mathrm{~mm} / \mathrm{s}$. The increase in the printing speed leads to a decrease in the part manufacturing duration but worsens the dimensional accuracy. High printing speeds reduce the degree of solidification of the bottom layers at the deposition of new layers. This can cause sliding processes between the successive deposited layers (mainly at the edges of the part) and thereby significant dimensional deviations.

Table 3. The influence of the printing speed on the mechanical properties of FFF-printed PLA.

\begin{tabular}{|c|c|c|c|c|c|c|c|c|c|}
\hline \multirow[b]{2}{*}{ Ref. } & \multicolumn{7}{|c|}{ FFF Process Parameters } & \multirow{2}{*}{$\begin{array}{l}\text { Mechanical Test } \\
\text { Settings }\end{array}$} & \multirow{2}{*}{$\begin{array}{l}\text { Results and } \\
\text { Conclusions }\end{array}$} \\
\hline & $\begin{array}{c}\mathrm{s}_{\mathrm{p}} \\
(\mathrm{mm} / \mathrm{s})\end{array}$ & $\begin{array}{c}\mathrm{t} \\
(\mathrm{mm})\end{array}$ & $\begin{array}{c}\mathrm{T}_{\mathrm{H}} \\
\left({ }^{\circ} \mathrm{C}\right)\end{array}$ & $\begin{array}{l}\mathbf{T}_{\mathbf{B}} \\
\left({ }^{\circ} \mathrm{C}\right)\end{array}$ & $\begin{array}{c}\text { B.O. } \\
(-)\end{array}$ & $\begin{array}{c}\theta \\
\left({ }^{\circ}\right)\end{array}$ & $\begin{array}{c}\text { Other } \\
\text { Parameters }\end{array}$ & & \\
\hline \multicolumn{10}{|c|}{$\begin{array}{l}\mathrm{s}_{\mathrm{p}} \text { - printing speed; } \mathrm{t} \text {-layer thickness (layer height); } \mathrm{T}_{\mathrm{H}} \text { - printing head (nozzle) temperature; } \mathrm{T}_{\mathrm{B}}-\text { build plate temperature; } \\
\text { B.O.-build orientation; } \theta \text {-raster angle; } \mathrm{d}_{\mathrm{f}}-\text { filament diameter; } \mathrm{d}_{\mathrm{n}} \text {-nozzle diameter; } \mathrm{W}_{\mathrm{L}}-\text { number of wall lines. }\end{array}$} \\
\hline [23] & $20-40$ & $0.10-0.20$ & 210 & - & $X Y$ & $45 /-45^{\circ}$ & $\begin{array}{c}\mathrm{d}_{\mathrm{f}}=1.75 \mathrm{~mm} ; \\
\mathrm{d}_{\mathrm{n}}=0.4 \mathrm{~mm} ; \\
20-80 \% \text { infill }\end{array}$ & $\begin{array}{c}\text { Tensile_ASTM } \\
\text { D638 }\end{array}$ & $\begin{array}{l}\text { Low increase in UTS } \\
\text { with the decrease in } \\
\text { printing speed. }\end{array}$ \\
\hline [26] & $20-80$ & $0.06-0.24$ & 210 & - & $\begin{array}{l}\text { YX; YZ; } \\
\text { ZY }\end{array}$ & $0^{\circ}$ & $\begin{array}{c}\mathrm{d}_{\mathrm{f}}=1.75 \mathrm{~mm} \\
\mathrm{~d}_{\mathrm{n}}=0.4 \mathrm{~mm} \\
100 \% \text { infill }\end{array}$ & $\begin{array}{c}\text { Tensile-ASTM } \\
\text { D638; } \\
\text { Bending- } \\
\text { ASTM } \\
\text { D790 }\end{array}$ & $\begin{array}{l}\text { The variation of UTS vs. } \\
\mathrm{s}_{\mathrm{p}} \text { is influenced by the } \\
\text { build orientation and } \\
\text { the layer thickness. }\end{array}$ \\
\hline [29] & $20-80$ & 0.40 & 215 & 55 & Horizontal & $\begin{array}{c}0^{\circ} ; 30^{\circ} ; 45^{\circ} \\
60^{\circ} ; 90^{\circ}\end{array}$ & $\begin{array}{c}100 \% \text { infill; } \\
W_{L}=2\end{array}$ & $\begin{array}{c}\text { Tensile_ASTM } \\
\text { D638; } \\
\mathrm{S}_{\mathrm{t}}=5 \mathrm{~mm} / \mathrm{min} \\
\end{array}$ & $\begin{array}{c}\text { Higher E and UTS } \\
\text { values for } \mathrm{s}_{\mathrm{p}}=20 \mathrm{~mm} / \mathrm{s} \text {. }\end{array}$ \\
\hline [30] & $12.5-50$ & 0.30 & $190-250$ & 60 & Vertical & - & $\begin{array}{c}\mathrm{d}_{\mathrm{f}}=2.85 \mathrm{~mm} ; \\
\mathrm{d}_{\mathrm{n}}=0.6 \mathrm{~mm} ; \\
\text { variable cooling }\end{array}$ & $\begin{array}{c}\text { Bending, } \\
\text { rectangular } \\
\text { hollow } \\
\text { cross-section; } \\
\mathrm{s}_{\mathrm{t}}=10 \mathrm{~mm} / \mathrm{min} \\
\end{array}$ & $\begin{array}{c}\text { For }_{\mathrm{H}}=210^{\circ} \mathrm{C} \text { highest } \\
\text { UFS }(56.3 \mathrm{MPa}) \text { at } \\
\mathrm{s}_{\mathrm{p}}=25 \mathrm{~mm} / \mathrm{s} \text {; high } \\
\text { influence of } \mathrm{s}_{\mathrm{p}} \text { on the } \\
\text { specimen mass. }\end{array}$ \\
\hline [54] & $70-170$ & 0.30 & 185 & - & Z & - & $\begin{array}{c}\mathrm{d}_{\mathrm{f}}=1.75 \mathrm{~mm} \\
100 \% \text { infill }\end{array}$ & $\begin{array}{c}\text { Tensile-ASTM } \\
\text { D638 IV; } \\
\mathrm{s}_{\mathrm{t}}=5 \mathrm{~mm} / \mathrm{min} \\
\end{array}$ & $\begin{array}{c}\text { Low variations of UTS } \\
\text { and E with } \\
\text { printing speed. }\end{array}$ \\
\hline [56] & $50-150$ & $0.10-0.40$ & 190-205 & - & - & - & $\begin{array}{c}\mathrm{d}_{\mathrm{f}}=1.75 \mathrm{~mm} \\
\mathrm{~d}_{\mathrm{n}}=0.4 \mathrm{~mm}\end{array}$ & $\begin{aligned} & \text { Tensile; } \\
& \mathrm{s}_{\mathrm{t}}=5 \mathrm{~mm} / \mathrm{min}\end{aligned}$ & $\begin{array}{c}\text { Higher UTS for } \\
\mathrm{s}_{\mathrm{p}}=80 \mathrm{~mm} / \mathrm{s} \text { and } \\
\mathrm{s}_{\mathrm{p}}=100 \mathrm{~mm} / \mathrm{s}\end{array}$ \\
\hline [59] & $40-80$ & $0.10-0.35$ & 220 & 25 & $\begin{array}{l}\alpha_{X Y}= \\
0^{\circ}-90^{\circ}\end{array}$ & - & $\begin{array}{c}\mathrm{d}_{\mathrm{f}}=1.75 \mathrm{~mm} \\
100 \% \text { infill }\end{array}$ & $\begin{array}{c}\text { Tensile-ASTM } \\
\text { D638 Type V } \\
\text { specimens } \\
\end{array}$ & $\begin{array}{l}\text { Higher E and UTS } \\
\text { values for low } \\
\text { printing speed. } \\
\end{array}$ \\
\hline [61] & $38-52$ & $0.20-0.30$ & 190 & 40 & - & $0^{\circ} ; 90^{\circ}$ & $\begin{array}{c}\mathrm{d}_{\mathrm{n}}=0.40 \mathrm{~mm} ; \\
40 \% \text { infill }\end{array}$ & $\begin{array}{c}\text { Bending- } \\
\text { ASTM D790; } \\
\mathrm{s}_{\mathrm{t}}=12 \mathrm{~mm} / \mathrm{min}\end{array}$ & $\begin{array}{l}\text { Higher flexural strength } \\
\text { for } \mathrm{s}_{\mathrm{p}}=38 \mathrm{~mm} / \mathrm{s} \text {. }\end{array}$ \\
\hline [62] & $25-75$ & $0.10-0.30$ & 210 & 60 & Vertical & - & $\begin{array}{l}\mathrm{d}_{\mathrm{n}}=0.40 \mathrm{~mm} ; \\
4 \text { FFF printers }\end{array}$ & $\begin{array}{l}\text { Bending, } \\
\text { rectangular } \\
\text { hollow } \\
\text { cross-section }\end{array}$ & $\begin{array}{l}\text { Higher UFS for } \\
\mathrm{s}_{\mathrm{p}}=25 \mathrm{~mm} / \mathrm{s}\end{array}$ \\
\hline
\end{tabular}


Table 3. Cont.

\begin{tabular}{|c|c|c|c|c|c|c|c|c|c|}
\hline \multirow[b]{2}{*}{ Ref. } & \multicolumn{7}{|c|}{ FFF Process Parameters } & \multirow{2}{*}{$\begin{array}{l}\text { Mechanical Test } \\
\text { Settings }\end{array}$} & \multirow{2}{*}{$\begin{array}{l}\text { Results and } \\
\text { Conclusions }\end{array}$} \\
\hline & $\underset{\substack{\mathrm{s}_{\mathrm{p}} \\
(\mathrm{mm} / \mathrm{s})}}{ }$ & $\begin{array}{c}\mathrm{t} \\
(\mathrm{mm})\end{array}$ & $\begin{array}{c}\mathrm{T}_{\mathrm{H}} \\
\left({ }^{\circ} \mathrm{C}\right)\end{array}$ & $\begin{array}{c}\mathrm{T}_{\mathrm{B}} \\
\left({ }^{\circ} \mathrm{C}\right)\end{array}$ & $\begin{array}{c}\text { B.O. } \\
(-)\end{array}$ & $\begin{array}{c}\theta \\
\left({ }^{\circ}\right)\end{array}$ & $\begin{array}{c}\text { Other } \\
\text { Parameters }\end{array}$ & & \\
\hline [65] & $30-90$ & $0.10-0.30$ & $210-230$ & $50-80$ & $X Y$ & $0^{\circ} / 90^{\circ}$ & $\begin{array}{c}\mathrm{d}_{\mathrm{f}}=1.75 \mathrm{~mm} \\
\mathrm{~d}_{\mathrm{n}}=0.4 \mathrm{~mm} \\
\mathrm{~W}_{\mathrm{L}}=2\end{array}$ & $\begin{array}{c}\text { Tensile-ISO } \\
527-2 ; \\
\mathrm{s}_{\mathrm{t}}=50 \mathrm{~mm} / \mathrm{min}\end{array}$ & $\begin{array}{l}\text { Low decrease in UTS } \\
\text { with the increase in the } \\
\text { printing speed. }\end{array}$ \\
\hline [73] & $20-60$ & 0.20 & 210 & 50 & - & - & $\mathrm{d}_{\mathrm{f}}=1.75 \mathrm{~mm}$ & $\begin{array}{c}\text { Tensile; } \mathrm{s}_{\mathrm{t}}=1 \\
\mathrm{~mm} / \mathrm{min}\end{array}$ & $\begin{array}{l}\text { Higher UTS for } \\
\mathrm{s}_{\mathrm{p}}=20 \mathrm{~mm} / \mathrm{s}\end{array}$ \\
\hline [80] & $40-50$ & 0.20 & $190-230$ & 50 & $X Y$ & $45^{\circ}$ & $\begin{array}{c}\mathrm{d}_{\mathrm{f}}=1.75 \mathrm{~mm} \\
\mathrm{~d}_{\mathrm{n}}=0.4 \mathrm{~mm} \\
100 \% \text { infill }\end{array}$ & $\begin{array}{l}\text { Tensile-ASTM } \\
\text { D638 Type IV } \\
\text { specimens }\end{array}$ & $\begin{array}{c}\text { Higher UTS values for } \\
\mathrm{s}_{\mathrm{p}}=50 \mathrm{~mm} / \mathrm{s} \\
\text { (except the } \\
\mathrm{T}_{\mathrm{H}}=230^{\circ} \mathrm{C} \text { specimens). }\end{array}$ \\
\hline [81] & $50-150$ & - & $190-210$ & - & Horizontal & - & $20-100 \%$ infill & $\begin{array}{c}\text { Tensile-ASTM } \\
\text { D638 Type V } \\
\text { specimens }\end{array}$ & $\begin{array}{c}\text { Highest UTS } \\
\text { (45.27 MPa) obtained for } \\
\mathrm{s}_{\mathrm{p}}=100 \mathrm{~mm} / \mathrm{s} \text { and } \\
\mathrm{T}_{\mathrm{H}}=210^{\circ} \mathrm{C} .\end{array}$ \\
\hline [82] & $60-100$ & $0.10-0.30$ & - & - & Horizontal & - & $60-100 \%$ infill & $\begin{array}{c}\text { Tensile_ASTM } \\
\text { D638; } \\
\text { Bending- } \\
\text { ASTM } \\
\text { D790 }\end{array}$ & $\begin{array}{l}\text { Infill density and } \\
\text { printing speed have the } \\
\text { highest influence on } \\
\text { UFS and UTS. }\end{array}$ \\
\hline [83] & $20-60$ & $0.08-0.28$ & $210-220$ & - & $X Y ; X Z$ & $\begin{array}{l}0^{\circ} / 90^{\circ} ; \\
30^{\circ} /-60^{\circ} \\
45^{\circ} /-45^{\circ}\end{array}$ & $\begin{array}{c}\mathrm{d}_{\mathrm{n}}=0.3-0.5 \mathrm{~mm} \\
80-100 \% \text { infill; } \\
\mathrm{W}_{\mathrm{L}}=2-4\end{array}$ & $\begin{array}{l}\text { Tensile-ASTM } \\
\quad \text { D638-I; } \\
\mathrm{s}_{\mathrm{t}}=5 \mathrm{~mm} / \mathrm{min}\end{array}$ & $\begin{array}{c}\text { Higher UTS for } \\
\mathrm{s}_{\mathrm{p}}=20 \mathrm{~mm} / \mathrm{s} \text {. The } \\
\text { optimum parameters for } \\
\text { UTS: } \mathrm{s}_{\mathrm{p}}=20 \mathrm{~mm} / \mathrm{s}, \\
\mathrm{T}_{\mathrm{H}}=220^{\circ} \mathrm{C}, \mathrm{XZ} \\
\text { orientation, } \\
30^{\circ} /-60^{\circ} \text { raster. }\end{array}$ \\
\hline [84] & $40-140$ & 0.10 & 210 & 50 & - & - & $\begin{array}{c}100 \% \text { infill; } \\
\mathrm{W}_{\mathrm{L}}=2 ; \\
\text { variable flow } \\
\text { rate }\end{array}$ & $\begin{array}{c}\text { Tensile-GB/T } \\
11997 \text { type-A } \\
\text { specimens; } \\
\mathrm{s}_{\mathrm{t}}=5 \mathrm{~mm} / \mathrm{min} \\
\end{array}$ & $\begin{array}{l}\text { Low influence of the } \\
\text { printing speed. High } \\
\text { influence of the } \\
\text { flow rate. }\end{array}$ \\
\hline [85] & $35-45$ & 0.20 & $180-220$ & 25 & $X Y$ & $45^{\circ} /-45^{\circ}$ & $\begin{array}{c}\mathrm{d}_{\mathrm{f}}=1.75 \mathrm{~mm} \\
\mathrm{~d}_{\mathrm{n}}=0.4 \mathrm{~mm} \\
20 \% \text { infill }\end{array}$ & $\begin{array}{c}\text { Tensile-ASTM } \\
\text { D638; } \\
\text { Bending- } \\
\text { ASTM D790; } \\
\text { Compression-- } \\
\text { ASTM D3410; } \\
\mathrm{s}_{\mathrm{t}}=5 \mathrm{~mm} / \mathrm{min}\end{array}$ & $\begin{array}{c}\text { Tensile: higher UTS for } \\
\mathrm{s}_{\mathrm{p}}=45 \mathrm{~mm} / \mathrm{s} \text { and } \\
\mathrm{s}_{\mathrm{p}}=40 \mathrm{~mm} / \mathrm{s} \text { at } \\
\mathrm{T}_{\mathrm{B}}=200-220{ }^{\circ} \mathrm{C} \text {. } \\
\text { Bending: higher UFS for } \\
\mathrm{s}_{\mathrm{p}}=45 \mathrm{~mm} / \mathrm{s} \text {. } \\
\text { Compression: higher } \\
\text { strength for } \\
\mathrm{s}_{\mathrm{p}}=45 \mathrm{~mm} / \mathrm{s} ;\end{array}$ \\
\hline [86] & $35-65$ & 0.10 & 200 & 60 & $X Y$ & $\begin{array}{c}45^{\circ} /-45^{\circ} \\
0^{\circ} / 90^{\circ}\end{array}$ & $\begin{array}{c}\mathrm{d}_{\mathrm{f}}=2.85 \mathrm{~mm} \\
100 \% \text { infill }\end{array}$ & $\begin{array}{c}\text { Tensile-ASTM } \\
\text { D638 }\end{array}$ & $\begin{array}{l}\text { Decrease in UTS with } \\
\text { the increase in the } \\
\text { printing speed. }\end{array}$ \\
\hline [87] & $50-100$ & $0.10-0.20$ & 210 & 60 & Vertical & - & $40-80 \%$ infill & $\begin{array}{c}\text { Bending, } \\
\text { circular hollow } \\
\text { cross-section } \\
\text { specimens } \\
\end{array}$ & $\begin{array}{l}\text { Higher UFS for low } \\
\text { printing speed and low } \\
\text { layer thickness. }\end{array}$ \\
\hline [88] & $30-40$ & - & 180-195 & - & - & $\begin{array}{l}45^{\circ} /-45^{\circ} \\
30^{\circ} /-60^{\circ} \\
0^{\circ} / 90^{\circ}\end{array}$ & - & $\begin{array}{c}\text { Tensile-ASTM } \\
\text { D638; } \\
\mathrm{s}_{\mathrm{t}}=5 \mathrm{~mm} / \mathrm{min} ; \\
\text { Bending- } \\
\text { ASTM D790; } \\
\mathrm{s}_{\mathrm{t}}=2 \mathrm{~mm} / \mathrm{min}\end{array}$ & $\begin{array}{c}\text { The optimum } \\
\text { parameters for tensile } \\
\text { test: } \mathrm{s}_{\mathrm{p}}=40 \mathrm{~mm} / \mathrm{s}, \\
\mathrm{T}_{\mathrm{H}}=180^{\circ} \\
\theta=30^{\circ} /-60^{\circ} . \\
\text { The optimum } \\
\text { parameters for bending } \\
\text { test: } \mathrm{s}_{\mathrm{p}}=30 \mathrm{~mm} / \mathrm{s}, \\
\mathrm{T}_{\mathrm{H}}=185^{\circ} \\
\theta=30^{\circ} /-60^{\circ} .\end{array}$ \\
\hline
\end{tabular}

\section{Printing Head Temperature and Build Plate Temperature}

The printing head temperature is one of the most studied process parameters. As revealed by Table 4 , the researchers selected printing head temperatures ranging from $175{ }^{\circ} \mathrm{C}$ to $275{ }^{\circ} \mathrm{C}$ for manufacturing of the PLA samples, but the most commonly analyzed temperatures were situated between $190-220^{\circ} \mathrm{C}$. These values correlate with the melting point of PLA $\left(160^{\circ} \mathrm{C}\right.$ up to $\left.180^{\circ} \mathrm{C}\right)$. The tendency to use lower temperatures is associated with the susceptibility of the PLA to thermal degradation at high temperatures and with economic issues (reduced energy consumption). At the same time, at low printing temperatures (below $180^{\circ} \mathrm{C}$, according to [34]), melting may not be complete and interlayer diffusion may not occur. Low diffusion between layers can cause delamination (peeling of layers). In [89] it is shown that at low printing temperatures the air gaps between raster lines are larger, which leads to reduced tensile strength. 
Table 4. The influence of the head temperature and build plate temperature on the mechanical properties of FFF-printed PLA.

\begin{tabular}{|c|c|c|c|c|c|c|c|c|c|}
\hline \multirow[b]{2}{*}{ Ref. } & \multicolumn{7}{|c|}{ FFF Process Parameters } & \multirow{2}{*}{$\begin{array}{l}\text { Mechanical Test } \\
\text { Settings }\end{array}$} & \multirow{2}{*}{$\begin{array}{l}\text { Results and } \\
\text { Conclusions }\end{array}$} \\
\hline & $\begin{array}{c}\mathrm{T}_{\mathrm{H}} \\
\left({ }^{\circ} \mathrm{C}\right)\end{array}$ & $\begin{array}{c}T_{B} \\
\left({ }^{\circ} \mathrm{C}\right) \\
\end{array}$ & $\begin{array}{c}\mathrm{t} \\
(\mathrm{mm})\end{array}$ & $\begin{array}{c}\mathrm{s}_{\mathrm{p}} \\
(\mathrm{mm} / \mathrm{s})\end{array}$ & $\begin{array}{l}\text { B.O. } \\
(-)\end{array}$ & $\begin{array}{c}\theta \\
\left({ }^{\circ}\right)\end{array}$ & $\begin{array}{c}\text { Other } \\
\text { Parameters }\end{array}$ & & \\
\hline \multicolumn{10}{|c|}{$\begin{array}{l}\mathrm{T}_{\mathrm{H}} \text { - printing head (nozzle) temperature; } \mathrm{T}_{\mathrm{B}}-\text { build plate temperature; } \mathrm{t} \text {-layer thickness (layer height); } \mathrm{s}_{\mathrm{p}}-\text { printing speed; } \\
\text { B.O.-build orientation; } \theta-\text { raster angle; } \mathrm{d}_{\mathrm{f}}-\text { filament diameter; } \mathrm{d}_{\mathrm{n}} \text {-nozzle diameter; } \mathrm{W}_{\mathrm{L}} \text {-number of wall lines. }\end{array}$} \\
\hline$[30]$ & $190-250$ & 60 & 0.30 & $12.5-50$ & Vertical & - & $\begin{array}{c}\mathrm{d}_{\mathrm{f}}=2.85 \mathrm{~mm} ; \\
\mathrm{d}_{\mathrm{n}}=0.6 \mathrm{~mm} ; \\
\text { variable cooling }\end{array}$ & $\begin{array}{l}\text { Bending, } \\
\text { rectangular } \\
\text { hollow } \\
\text { cross-section; } \\
\mathrm{s}_{\mathrm{t}}=10 \mathrm{~mm} / \mathrm{min}\end{array}$ & $\begin{array}{c}\text { Increase in ultimate } \\
\text { flexural strength and } \\
\text { specimen mass with the } \\
\text { printing } \\
\text { head temperature. }\end{array}$ \\
\hline [33] & $190-230$ & $45-105$ & - & 50 & - & $\begin{array}{l}0^{\circ} / 90^{\circ} \\
15^{\circ} / 75^{\circ} \\
30^{\circ} / 60^{\circ} \\
45^{\circ} / 45^{\circ}\end{array}$ & $\begin{array}{c}\mathrm{d}_{\mathrm{f}}=2.85 \mathrm{~mm} \\
100 \% \text { infill }\end{array}$ & $\begin{array}{c}\text { Tensile-ASTM } \\
\text { D638; } \\
\text { st = } 5 \mathrm{~mm} / \mathrm{min} \\
\text { Bending- } \\
\text { ASTM D790; } \\
\text { Impact-ASTM } \\
\text { D256 }\end{array}$ & $\begin{array}{l}\text { Mechanical parameters } \\
\text { increase with } \mathrm{T}_{\mathrm{B}} \text {. The } \\
\text { influence of } \mathrm{T}_{\mathrm{H}} \text { is lower } \\
\text { compared to the } \\
\text { influence } \mathrm{T}_{\mathrm{B}} \text {. }\end{array}$ \\
\hline [34] & $180-240$ & - & 0.10 & 60 & Horizontal & - & $\begin{array}{l}\mathrm{d}_{\mathrm{f}}=1.75 \mathrm{~mm} ; \\
\text { annealing }\end{array}$ & $\begin{array}{c}\text { Tensile- } \\
\text { ISO527; } \\
\mathrm{s}_{\mathrm{t}}=5 \mathrm{~mm} / \mathrm{min} \\
\end{array}$ & $\begin{array}{c}\text { Increase in UTS and E } \\
\text { with } T_{H} \text { for specimens } \\
\text { without annealing. }\end{array}$ \\
\hline [51] & $190-220$ & 60 & $0.06-0.35$ & 60 & $X Y$ & $\begin{array}{r}0^{\circ} ; 90^{\circ} \\
45 /-45^{\circ}\end{array}$ & $\begin{array}{c}\mathrm{d}_{\mathrm{f}}=1.75 \mathrm{~mm} \\
\mathrm{~d}_{\mathrm{n}}=0.4 \mathrm{~mm} \\
\mathrm{~W}_{\mathrm{L}}=2\end{array}$ & $\begin{array}{l}\text { Tensile-ASTM } \\
\text { D638-I } \\
\text { specimens; } \\
\mathrm{s}_{\mathrm{t}}=5 \mathrm{~mm} / \mathrm{min}\end{array}$ & $\begin{array}{l}\text { Highest UTS values for } \\
\mathrm{T}_{\mathrm{H}}=220^{\circ} \mathrm{C} \text { and } \\
\mathrm{T}_{\mathrm{H}}=205^{\circ} \mathrm{C} \text {. High } \\
\text { variation of UTS vs. } \mathrm{T}_{\mathrm{H}} \\
\text { for } \theta=90^{\circ} \text {. }\end{array}$ \\
\hline [54] & $175-205$ & - & 0.30 & 90 & $\mathrm{Z}$ & - & $\begin{array}{c}\mathrm{d}_{\mathrm{f}}=1.75 \mathrm{~mm} \\
100 \% \text { infill }\end{array}$ & $\begin{array}{l}\text { Tensile-ASTM } \\
\text { D638 Type IV; } \\
\mathrm{S}_{\mathrm{t}}=5 \mathrm{~mm} / \mathrm{min}\end{array}$ & $\begin{array}{c}\text { Highest UTS } \\
(43.79 \mathrm{MPa}) \text { at } \\
\mathrm{T}_{\mathrm{H}}=205^{\circ} \mathrm{C} \text {. Approx. } \\
35 \% \text { increase in UTS for } \\
\mathrm{T}_{\mathrm{H}}=205^{\circ} \mathrm{C} \text {, compared } \\
\text { to } \mathrm{T}_{\mathrm{H}}=175^{\circ} \mathrm{C} \text {. }\end{array}$ \\
\hline [55] & $190-210$ & - & $0.20-0.40$ & 50 & Horizontal & - & $\begin{array}{c}\mathrm{d}_{\mathrm{f}}=2.85 \mathrm{~mm} ; \\
20-100 \% \text { infill; } \\
\mathrm{W}_{\mathrm{L}}=2\end{array}$ & $\begin{array}{l}\text { Tensile-ASTM } \\
\text { D638; increased } \\
\text { specimen } \\
\text { thickness; } \\
\mathrm{S}_{\mathrm{t}}=5 \mathrm{~mm} / \mathrm{min}\end{array}$ & $\begin{array}{l}\text { Highest UTS for } \\
\mathrm{T}_{\mathrm{H}}=210^{\circ} \mathrm{C} \text { and } \\
\mathrm{T}_{\mathrm{H}}=200^{\circ} \mathrm{C} .\end{array}$ \\
\hline [56] & 190-205 & - & $0.10-0.40$ & $50-150$ & Horizontal & - & $\begin{array}{c}\mathrm{d}_{\mathrm{f}}=1.75 \mathrm{~mm} \\
\mathrm{~d}_{\mathrm{n}}=0.4 \mathrm{~mm} \\
\text { cooling fan }\end{array}$ & $\begin{array}{c}\text { Tensile test; } \\
\mathrm{s}_{\mathrm{t}}=5 \mathrm{~mm} / \mathrm{min}\end{array}$ & $\begin{array}{l}\text { Higher UTS obtained for } \\
\mathrm{T}_{\mathrm{H}}=210^{\circ} \mathrm{C} \text { and active } \\
\text { cooling fan; higher } \mathrm{T}_{\mathrm{H}} \\
\text { recommended for high } \\
\text { layer thickness. }\end{array}$ \\
\hline$[65]$ & $210-230$ & $50-80$ & $0.10-0.30$ & $30-90$ & $X Y$ & $0^{\circ} / 90^{\circ}$ & $\begin{array}{c}\mathrm{d}_{\mathrm{n}}=0.4 \mathrm{~mm} ; \\
\mathrm{d}_{\mathrm{f}}=1.75 \mathrm{~mm} ; \\
100 \% \text { infill; } \\
\mathrm{W}_{\mathrm{L}}=2\end{array}$ & $\begin{array}{c}\text { Tensile-ISO } \\
527-2 ; \\
\mathrm{s}_{\mathrm{t}}=50 \mathrm{~mm} / \mathrm{min}\end{array}$ & $\begin{array}{l}\text { Low increase in UTS } \\
\text { with the increase in } \mathrm{T}_{\mathrm{H}} \\
\text { and decrease in } \mathrm{T}_{\mathrm{B}} \text {. }\end{array}$ \\
\hline [73] & $200-230$ & $50-70$ & 0.20 & 20 & - & - & $\mathrm{d}_{\mathrm{f}}=1.75 \mathrm{~mm}$ & $\begin{aligned} & \text { Tensile; } \\
& \mathrm{s}_{\mathrm{t}}=1 \mathrm{~mm} / \mathrm{min}\end{aligned}$ & $\begin{array}{c}\text { Highest UTS }(62 \mathrm{MPa}) \\
\text { for } \mathrm{T}_{\mathrm{H}}=220^{\circ} \mathrm{C} ; \\
\text { Low variation of UTS } \\
\text { vs. } \mathrm{T}_{\mathrm{B} .}\end{array}$ \\
\hline [74] & $195-230$ & 60 & $0.05-0.20$ & 60 & $\begin{array}{l}\beta_{Y Z}= \\
0^{\circ}-90^{\circ}\end{array}$ & - & $\begin{array}{c}\mathrm{d}_{\mathrm{f}}=1.75 \mathrm{~mm} ; \\
\mathrm{d}_{\mathrm{n}}=0.4 \mathrm{~mm}\end{array}$ & $\begin{array}{c}\text { Tensile-ISO } \\
527-2 ; \\
\mathrm{s}_{\mathrm{t}}=2 \mathrm{~mm} / \mathrm{min} \\
\end{array}$ & $\begin{array}{l}\text { Higher UTS for } \\
\mathrm{T}_{\mathrm{B}}=210-215^{\circ} \mathrm{C} .\end{array}$ \\
\hline [80] & $190-230$ & 50 & 0.20 & $40-50$ & $X Y$ & $45^{\circ}$ & $\begin{array}{c}\mathrm{d}_{\mathrm{f}}=1.75 \mathrm{~mm} ; \\
\mathrm{d}_{\mathrm{n}}=0.4 \mathrm{~mm} ; \\
100 \% \text { infill; } \\
\mathrm{W}_{\mathrm{L}}=3\end{array}$ & $\begin{array}{c}\text { Tensile-ASTM } \\
\text { D638 Type IV } \\
\text { specimens }\end{array}$ & $\begin{array}{l}\text { Approx. } 20 \% \text { increase in } \\
\text { UTS for } \mathrm{T}_{\mathrm{H}}=210^{\circ} \mathrm{C} \text {, } \\
\text { compared to } \\
\mathrm{T}_{\mathrm{H}}=190^{\circ} \mathrm{C} \text {. }\end{array}$ \\
\hline [81] & $190-210$ & - & - & $50-150$ & Horizontal & - & $20-100 \%$ infill & $\begin{array}{c}\text { Tensile-ASTM } \\
\text { D638 Type V } \\
\text { specimens }\end{array}$ & $\begin{array}{c}\text { Highest UTS } \\
\text { (45.27 MPa) obtained for } \\
\mathrm{s}_{\mathrm{p}}=100 \mathrm{~mm} / \mathrm{s} \text { and } \\
\mathrm{T}_{\mathrm{H}}=210^{\circ} \mathrm{C} .\end{array}$ \\
\hline [83] & $210-220$ & - & $0.08-0.28$ & $20-60$ & $X Y ; X Z$ & $\begin{array}{c}0^{\circ} / 90^{\circ} \\
30^{\circ} /-60^{\circ} \\
45^{\circ} /-45^{\circ}\end{array}$ & $\begin{array}{c}\mathrm{d}_{\mathrm{f}}=1.75 \mathrm{~mm} ; \\
\mathrm{d}_{\mathrm{n}}=0.3-0.5 \mathrm{~mm} ; \\
80-100 \% \text { infill; } \\
\mathrm{W}_{\mathrm{L}}=2-4\end{array}$ & $\begin{array}{l}\text { Tensile-ASTM } \\
\text { D638-I; } \\
\mathrm{s}_{\mathrm{t}}=5 \mathrm{~mm} / \mathrm{min}\end{array}$ & $\begin{array}{l}\text { Higher UTS for } \\
\mathrm{T}_{\mathrm{H}}=220^{\circ} \mathrm{C} .\end{array}$ \\
\hline [85] & $180-220$ & 25 & 0.20 & $35-45$ & $X Y$ & $45^{\circ} /-45^{\circ}$ & $\begin{array}{c}\mathrm{d}_{\mathrm{f}}=1.75 \mathrm{~mm} \\
\mathrm{~d}_{\mathrm{n}}=0.4 \mathrm{~mm} \\
20 \% \text { infill }\end{array}$ & $\begin{array}{c}\text { Tensile-ASTM } \\
\text { D638; } \\
\text { Bending- } \\
\text { ASTM D790; } \\
\text { Compression- } \\
\text { ASTM } \\
\text { D3410 }\end{array}$ & $\begin{array}{c}\text { Higher UTS for } \\
\mathrm{T}_{\mathrm{H}}=220^{\circ} \mathrm{C} \text {; Higher } \\
\text { compressive strength for } \\
\mathrm{T}_{\mathrm{H}}=190-220^{\circ} \mathrm{C} \text {; Higher } \\
\text { bending strength for } \\
\mathrm{T}_{\mathrm{H}}=190-210^{\circ} \mathrm{C} .\end{array}$ \\
\hline
\end{tabular}


Table 4. Cont.

\begin{tabular}{|c|c|c|c|c|c|c|c|c|c|}
\hline \multirow[b]{2}{*}{ Ref. } & \multicolumn{7}{|c|}{ FFF Process Parameters } & \multirow{2}{*}{$\begin{array}{l}\text { Mechanical Test } \\
\text { Settings }\end{array}$} & \multirow{2}{*}{$\begin{array}{l}\text { Results and } \\
\text { Conclusions }\end{array}$} \\
\hline & $\begin{array}{c}\mathrm{T}_{\mathrm{H}} \\
\left({ }^{\circ} \mathrm{C}\right)\end{array}$ & $\begin{array}{c}\mathrm{T}_{\mathrm{B}} \\
\left({ }^{\circ} \mathrm{C}\right)\end{array}$ & $\begin{array}{c}\mathrm{t} \\
(\mathrm{mm})\end{array}$ & $\begin{array}{c}\mathrm{s}_{\mathrm{p}} \\
(\mathrm{mm} / \mathrm{s})\end{array}$ & $\begin{array}{l}\text { B.O. } \\
(-)\end{array}$ & $\begin{array}{c}\theta \\
\left({ }^{\circ}\right)\end{array}$ & $\begin{array}{c}\text { Other } \\
\text { Parameters }\end{array}$ & & \\
\hline [90] & $190-210$ & 55 & 0.35 & 40 & Horizontal & $0^{\circ} ; 45^{\circ} ; 90^{\circ}$ & $\begin{array}{c}\mathrm{d}_{\mathrm{f}}=2.85 \mathrm{~mm} \\
\mathrm{~d}_{\mathrm{n}}=0.4 \mathrm{~mm} \\
\mathrm{~W}_{\mathrm{L}}=2\end{array}$ & $\begin{array}{l}\text { Tensile-ASTM } \\
\text { D638-10-I; } \\
\mathrm{s}_{\mathrm{t}}=5 \mathrm{~mm} / \mathrm{min}\end{array}$ & $\begin{array}{l}\text { Higher UTS and E for } \\
\mathrm{T}_{\mathrm{H}}=210^{\circ} \mathrm{C} \text { (for all } \\
\text { raster). Highest UTS } \\
(56.2 \mathrm{MPa} \text { ) for } \\
\text { specimens with } \\
\mathrm{T}_{\mathrm{H}}=210^{\circ} \mathrm{C} \text { and } \theta=0^{\circ} .\end{array}$ \\
\hline [91] & $180-210$ & 60 & 0.20 & 50 & $X Y$ & $45 /-45^{\circ}$ & $\begin{array}{c}\mathrm{d}_{\mathrm{n}}=0.4 \mathrm{~mm} ; \\
100 \% \text { infill; } \\
70-160 \% \text { flow }\end{array}$ & $\begin{array}{c}\text { Tensile-ISO } \\
527-2\end{array}$ & $\begin{array}{c}\text { The variation of tensile } \\
\text { load vs. temperature is } \\
\text { influenced by the } \\
\text { flow rate. }\end{array}$ \\
\hline [92] & 210 & $40-80$ & 0.20 & - & Horizontal & $45 /-45^{\circ}$ & $\begin{array}{c}\mathrm{d}_{\mathrm{f}}=1.75 \mathrm{~mm} \\
\mathrm{~d}_{\mathrm{n}}=0.4 \mathrm{~mm}\end{array}$ & $\begin{array}{c}\text { Tensile-ASTM } \\
\text { D638 Type IV } \\
\text { specimens }\end{array}$ & $\begin{array}{l}\text { Higher strength for } \\
\text { specimens printed } \\
\text { inside of a } \\
\text { heated chamber. }\end{array}$ \\
\hline [93] & 195-255 & 55 & 0.30 & 45 & $X Y$ & $0^{\circ}$ & $\begin{array}{c}\mathrm{d}_{\mathrm{f}}=1.75 \mathrm{~mm} ; \\
\mathrm{d}_{\mathrm{n}}=0.5 \mathrm{~mm} ; \\
100 \% \text { infill; } \\
\text { annealing }\end{array}$ & $\begin{array}{c}\text { Tensile-ISO } \\
\text { 527; } \\
\text { Bending-EN } \\
\text { ISO } \\
\text { 178:2011 } \\
\end{array}$ & $\begin{array}{l}\text { Higher UTS and UFS for } \\
\mathrm{T}_{\mathrm{H}}=235-255^{\circ} \mathrm{C} .\end{array}$ \\
\hline [94] & $180-230$ & 70-110 & 0.30 & 40 & $\mathrm{YZ}$ & $0^{\circ} / 90^{\circ}$ & $\begin{array}{c}\mathrm{d}_{\mathrm{f}}=1.75 \mathrm{~mm} \\
\mathrm{~d}_{\mathrm{n}}=0.4 \mathrm{~mm} \\
99 \% \text { infill }\end{array}$ & $\begin{array}{c}\text { Tensile-ASTM } \\
\text { D368 Type V } \\
\text { specimens }\end{array}$ & $\begin{array}{c}\text { Highest UTS }(76.5 \mathrm{MPa}) \\
\text { for } \mathrm{T}_{\mathrm{H}}=200^{\circ} \mathrm{C} \text { and } \\
\mathrm{T}_{\mathrm{B}}=70^{\circ} \mathrm{C} \text {. Lowest UTS } \\
(69 \mathrm{MPa}) \text { for } \mathrm{T}_{\mathrm{H}}=180^{\circ} \mathrm{C} \\
\text { and } \mathrm{T}_{\mathrm{B}}=110^{\circ} \mathrm{C} .\end{array}$ \\
\hline [95] & $210-230$ & 70 & 0.20 & 40 & $X Y$ & $45^{\circ} /-45^{\circ}$ & $\begin{array}{c}\mathrm{d}_{\mathrm{f}}=1.75 \mathrm{~mm} ; \\
100 \% \text { infill }\end{array}$ & $\begin{array}{c}\text { Tensile-ASTM } \\
\text { D368 Type IV } \\
\text { specimens; } \\
\mathrm{s}_{\mathrm{t}}=1 \mathrm{~mm} / \mathrm{min}\end{array}$ & $\begin{array}{c}\text { Highest UTS }(53 \mathrm{MPa}) \\
\text { and } \mathrm{E}(2.5 \mathrm{GPa}) \text { for } \\
\mathrm{T}_{\mathrm{H}}=220^{\circ} \mathrm{C} \text {. Lowest UTS } \\
(47 \mathrm{MPa}) \text { and } \mathrm{E}(2.2 \mathrm{GPa}) \\
\text { for } \mathrm{T}_{\mathrm{H}}=230^{\circ} \mathrm{C} \text {. }\end{array}$ \\
\hline
\end{tabular}

Higher printing head temperatures can provide better interlayer diffusion and higher mechanical properties, but also a slip of the deposited material, affecting the dimensional accuracy of the components. In [34] it is shown that the use of printing temperatures above $240{ }^{\circ} \mathrm{C}$ causes an unsteady flow of material from the printing head nozzle.

The build plate temperature is generally set in the range of $50-60^{\circ} \mathrm{C}$. In open-space $3 \mathrm{D}$ printers, the uniformity of the build plate temperature is difficult to achieve because of the heat flows. In general, in the central areas of the build plate the temperature is higher compared to the peripheral areas. This disadvantage is mitigated for the printers by closed work space and controlled heat flow. In [60] it is shown that the influence of the heat flux on the ultimate tensile strength is low when the specimens are printed horizontally and high when the specimens are printed vertically.

Considering both temperature-related parameters-the printing head temperature and the build plate temperature, respectively-it is shown that the influence of the printing head temperature on the mechanical properties is lower compared to the influence of the build plate temperature [33].

The importance of temperature profile monitoring during the FFF-printing process by using specific devices (infrared camera, thermocouples) and the development of numerical heat transfer models is highlighted in [96].

\section{Build Orientation of the Specimens}

The placement of the 3D model onto the building space of the printer is one of the main factors that determine the anisotropic behavior of PLA FFF-printed parts. In this regard, high differences were found between the mechanical behavior along the $\mathrm{x}$ and $\mathrm{y}$ axes (axes situated in the plane of the build plate-Figure 2) and the mechanical behavior along the vertical z-axis. Variations of mechanical properties for the parts rotated with various angles to the reference system must also be included in the analysis. 


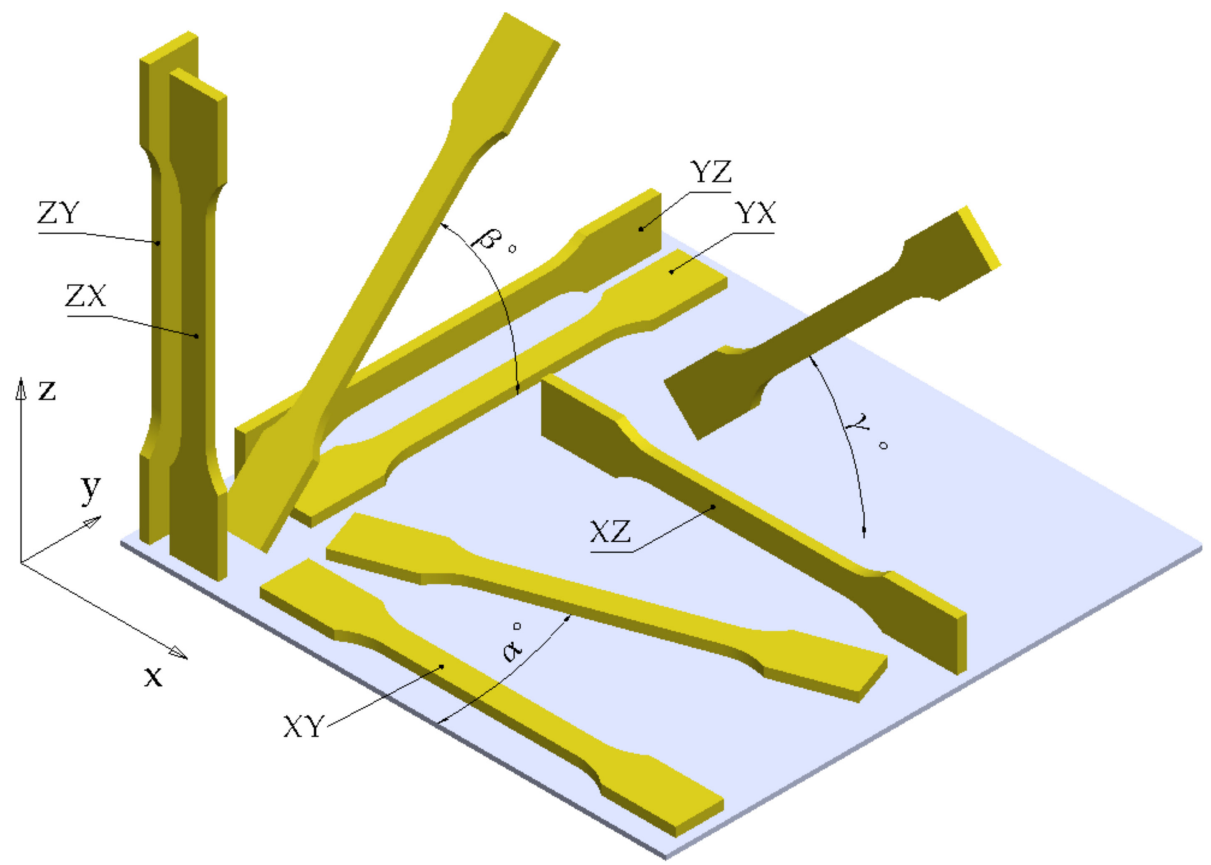

Figure 2. Notation of different build orientations.

In the ISO/ASTM 52921:2013 standard [17] the notation of the orthogonal orientation (non-rotated) of a prismatic part relative to the printer reference system is done by combinations of three letters: the first letter of the notation represents the axis parallel to the longest characteristic dimension of the part, the second letter represents the axis parallel to the second-longest characteristic dimension of the part and the third letter represents the axis parallel to the third characteristic dimension. If the part has a symmetry plane (as in the case of dog-bone tensile specimens), a simplified notation consisting of the first two letters may be used.

Figure 2 shows the notation of the positioning of an ISO 527-2:2012 Type 1A tensile specimen. The first characteristic dimension is the length of the specimen and the second characteristic dimension is the width of the specimen. The necessity of using standardized notations for build orientation results from the analysis of the articles published so far (Table 5). In several articles, $X Y$ and $Y X$ build orientations are referred to as "flat build orientations", $X Z$ and $Y Z$ build orientations are referred to as "on-edge build orientations" and $\mathrm{ZX}$ and $\mathrm{ZY}$ build orientations are referred to as "upright build orientations". The use of the term "flat build orientation", without graphic detail, does not clearly indicate whether $\mathrm{XY}$ or $\mathrm{YX}$ build orientation is used. The ambiguity is amplified in the cases where rotated specimens relative to the orthogonal orientation are used. In this paper we propose the use of angles $\alpha, \beta$ and $\gamma$ for describing rotations in the $\mathrm{xy}, \mathrm{yz}$ and $\mathrm{zx}$ planes. To define the angle of rotation relative to an orthogonal orientation, indices will be used (angle $\alpha_{X Z}$ defines a specimen rotated by $\alpha^{\circ}$ in the xy plane relative to the base orientation $X Z$, angle $\alpha_{X Z}=0^{\circ}$ represents the $X Z$ orthogonal orientation and $\alpha_{X Z}=90^{\circ}$ represents the $Y Z$ orientation). For a comparative analysis, the notations from Figure 2 were used for the papers listed in Table 5. For some papers, where it was not possible to unambiguously identify the build orientation, the notations given by the authors were maintained. 
Table 5. The influence of the build orientation and the printing orientation angle on the mechanical properties of FFF-printed PLA.

\begin{tabular}{|c|c|c|c|c|c|c|c|c|c|}
\hline \multirow[b]{2}{*}{ Ref. } & \multicolumn{7}{|c|}{ FFF Process Parameters } & \multirow{2}{*}{$\begin{array}{l}\text { Mechanical } \\
\text { Test Settings }\end{array}$} & \multirow{2}{*}{$\begin{array}{l}\text { Results and } \\
\text { Conclusions }\end{array}$} \\
\hline & $\begin{array}{c}\text { B.O. } \\
(-)\end{array}$ & $\begin{array}{c}\mathbf{t} \\
(\mathrm{mm})\end{array}$ & $\begin{array}{c}\mathrm{s}_{\mathrm{p}} \\
(\mathrm{mm} / \mathrm{s})\end{array}$ & $\begin{array}{c}\mathrm{T}_{\mathrm{H}} \\
\left({ }^{\circ} \mathrm{C}\right)\end{array}$ & $\begin{array}{c}\mathrm{T}_{\mathrm{B}} \\
\left({ }^{\circ} \mathrm{C}\right)\end{array}$ & $\begin{array}{c}\theta \\
\left({ }^{\circ}\right) \\
\end{array}$ & $\begin{array}{c}\text { Other } \\
\text { Parameters } \\
\end{array}$ & & \\
\hline \multicolumn{10}{|c|}{$\begin{array}{l}\text { B.O.-build orientation; } \mathrm{t}-\text { layer thickness (layer height); } \mathrm{s}_{\mathrm{p}}-\text { printing speed; } \mathrm{T}_{\mathrm{H}} \text {-printing head (nozzle) temperature; } \\
\mathrm{T}_{\mathrm{B}} \text { - build plate temperature; } \theta \text {-raster angle; } \mathrm{d}_{\mathrm{f}} \text {-filament diameter; } \mathrm{d}_{\mathrm{n}} \text {-nozzle diameter; } \mathrm{W}_{\mathrm{L}} \text {-number of wall lines. }\end{array}$} \\
\hline [26] & $\begin{array}{l}\text { YX; YZ; } \\
\text { ZY }\end{array}$ & $0.06-0.24$ & $20-80$ & 210 & - & $0^{\circ}$ & $\begin{array}{c}\mathrm{d}_{\mathrm{f}}=1.75 \mathrm{~mm} \\
\mathrm{~d}_{\mathrm{n}}=0.4 \mathrm{~mm} \\
100 \% \text { infill }\end{array}$ & $\begin{array}{c}\text { Tensile-ASTM } \\
\text { D638; } \\
\text { Bending- } \\
\text { ASTM } \\
\text { D790 }\end{array}$ & $\begin{array}{l}\text { High variation of UTS } \\
\text { and UFS. Highest values } \\
\text { for YX and } \\
\text { YZ specimens. }\end{array}$ \\
\hline [27] & $\begin{array}{l}X Y ; X Z ; \\
Z X\end{array}$ & 0.20 & - & - & - & $45^{\circ} /-45^{\circ}$ & $\begin{array}{l}50-100 \% \\
\text { infill }\end{array}$ & $\begin{array}{c}\text { Tensile-ISO } \\
527-2 ; \\
\mathrm{s}_{\mathrm{t}}=10 \mathrm{~mm} / \mathrm{min}\end{array}$ & $\begin{array}{l}\text { Highest UTS (56.5 MPa) } \\
\text { for flat XY specimens at } \\
100 \% \text { infill. } 13 \% \text { and } 37 \% \\
\text { decrease in UTS for XZ } \\
\text { and ZX specimens. }\end{array}$ \\
\hline [28] & $X Y ; Z X$ & $0.06-0.50$ & $30-200$ & $175-230$ & - & - & $\begin{array}{l}\mathrm{d}_{\mathrm{f}}=1.75 \mathrm{~mm} ; \\
\mathrm{d}_{\mathrm{n}}=0.5 \mathrm{~mm} ; \\
100 \% \text { infill; } \\
\text { variable flow }\end{array}$ & $\begin{array}{l}\text { Tensile-ASTM } \\
\text { D638 Type I vs. } \\
\text { Type IV }\end{array}$ & $\begin{array}{c}\text { UTS for ZX specimens is } \\
47.9 \% \text { lower compared } \\
\text { to UTS for } \\
\text { XY specimens. }\end{array}$ \\
\hline [54] & $X ; Y ; Z$ & 0.30 & 90 & 185 & - & - & $\begin{array}{c}\mathrm{d}_{\mathrm{f}}=1.75 \mathrm{~mm} \\
100 \% \text { infill }\end{array}$ & $\begin{array}{l}\text { Tensile-ASTM } \\
\text { D638 IV; } \\
\mathrm{s}_{\mathrm{t}}=5 \mathrm{~mm} / \mathrm{min} \\
\end{array}$ & $\begin{array}{l}\text { Low variation of UTS } \\
\text { with build orientation. }\end{array}$ \\
\hline$[60]$ & $\begin{array}{l}\text { Horizontal; } \\
\text { vertical }\end{array}$ & $0.05-0.40$ & 60 & 200 & - & - & $\begin{array}{l}\mathrm{d}_{\mathrm{f}}=1.75 \mathrm{~mm} ; \\
\quad 60 \% \text { infill }\end{array}$ & Tensile & $\begin{array}{c}\text { UTS for vertical } \\
\text { specimens } 50 \% \text { lower } \\
\text { than UTS for } \\
\text { horizontal specimens. }\end{array}$ \\
\hline$[72]$ & $\begin{array}{l}X Y ; X Z ; \\
Z X\end{array}$ & 0.10 & 30 & 200 & 50 & $45^{\circ} /-45^{\circ}$ & $\begin{array}{c}\mathrm{d}_{\mathrm{f}}=1.75 \mathrm{~mm} \\
\mathrm{~d}_{\mathrm{n}}=0.4 \mathrm{~mm} \\
20 \% \text { infill }\end{array}$ & $\begin{array}{l}\text { Tensile-ASTM } \\
\text { D638; } \\
\mathrm{s}_{\mathrm{t}}=5 \mathrm{~mm} / \mathrm{min}\end{array}$ & $\begin{array}{l}\text { Higher UTS (38.47 MPa) } \\
\text { for XY specimens } \\
\text { compared to XZ } \\
(30.10 \mathrm{MPa}) \text { and } Z X \\
(27.63 \mathrm{MPa}) \text { specimens. }\end{array}$ \\
\hline [83] & $X Y ; X Z$ & $0.08-0.28$ & $20-60$ & $210-220$ & - & $\begin{array}{l}0^{\circ} / 90^{\circ} ; \\
30^{\circ} /-60^{\circ} ; \\
45^{\circ} /-45^{\circ}\end{array}$ & $\begin{array}{c}\mathrm{d}_{\mathrm{n}}=0.3-0.5 \mathrm{~mm} ; \\
80-100 \% \text { infill; } \\
\mathrm{W}_{\mathrm{L}}=2-4\end{array}$ & $\begin{array}{l}\text { Tensile-ASTM } \\
\text { D638-I; } \\
\mathrm{s}_{\mathrm{t}}=5 \mathrm{~mm} / \mathrm{min} \\
\end{array}$ & $\begin{array}{l}\text { Higher UTS for } \\
\text { XZ specimens. }\end{array}$ \\
\hline [97] & $\begin{array}{l}\mathrm{XY} ; \mathrm{XZ} ; \\
\mathrm{ZX}\end{array}$ & 0.40 & 3 & 220 & - & - & $\begin{array}{c}\mathrm{d}_{\mathrm{n}}=0.4 \mathrm{~mm} \\
100 \% \text { infill }\end{array}$ & $\begin{array}{c}\text { Tensile-ASTM } \\
\text { D638 } \\
\end{array}$ & $\begin{array}{l}\text { Highest values of E and } \\
\text { UTS for XZ specimens. }\end{array}$ \\
\hline [98] & $\begin{array}{l}X Y ; X Z ; \\
Z X\end{array}$ & 0.20 & 60 & 210 & 45 & $45^{\circ} /-45^{\circ}$ & $\begin{array}{c}\mathrm{d}_{\mathrm{f}}=1.75 \mathrm{~mm} \\
\mathrm{~d}_{\mathrm{n}}=0.4 \mathrm{~mm} \\
\mathrm{~W}_{\mathrm{L}}=2\end{array}$ & $\begin{array}{l}\text { Tensile-ASTM } \\
\text { D638 Type I } \\
\text { specimens }\end{array}$ & $\begin{array}{l}\text { Highest values of UTS } \\
\text { (57.58 MPa) and E } \\
\text { (2571 MPa) for XY } \\
\text { specimens. Low value of } \\
\text { UTS (23.75 MPa) for } \\
\text { ZX specimens. }\end{array}$ \\
\hline [99] & $\begin{array}{l}X Y ; X Z ; \\
\quad Z X\end{array}$ & 0.18 & 80 & - & - & - & $\begin{array}{l}\mathrm{d}_{\mathrm{f}}=1.75 \mathrm{~mm} \\
20-100 \% \text { infill }\end{array}$ & $\begin{array}{l}\text { Tensile-ASTM } \\
\text { D638; } \\
\mathrm{s}_{\mathrm{t}}=5 \mathrm{~mm} / \mathrm{min}\end{array}$ & $\begin{array}{c}\text { Yield stress for } 100 \% \\
\text { infill: XY } \\
\text { specimens-41.66 MPa, } \\
\text { XZ } \\
\text { specimens_-48.53 MPa, } \\
\text { ZX } \\
\text { specimens-24.20 MPa. } \\
\text { Similar variation for } \\
\text { lower infill density. }\end{array}$ \\
\hline [100] & $\begin{array}{l}X Y ; X Z ; \\
Z X\end{array}$ & 0.20 & 50 & 215 & 60 & $0^{\circ} ; 45^{\circ} ; 90^{\circ}$ & $\begin{array}{c}\mathrm{d}_{\mathrm{f}}=1.75 \mathrm{~mm} ; \\
\mathrm{d}_{\mathrm{n}}=0.4 \mathrm{~mm} ; \\
100 \% \text { infill; } \\
\mathrm{W}_{\mathrm{L}}=2\end{array}$ & $\begin{array}{l}\text { Tensile-ASTM } \\
\text { D638; } \\
\mathrm{s}_{\mathrm{t}}=5 \mathrm{~mm} / \mathrm{min}\end{array}$ & $\begin{array}{c}\text { Higher UTS } \\
(34.45-35.47 \mathrm{MPa}) \text { for } \\
\text { XZ specimens. Low UTS } \\
\text { for XY and ZX } \\
\text { specimens. The } \\
\text { variations are influenced } \\
\text { by the raster. }\end{array}$ \\
\hline [57] & $\begin{array}{c}\alpha_{X Y}=0^{\circ}- \\
60^{\circ}\end{array}$ & $0.10-0.30$ & 50 & 210 & 60 & - & $\begin{array}{c}\mathrm{d}_{\mathrm{f}}=1.75 \mathrm{~mm} ; \\
\mathrm{d}_{\mathrm{n}}=0.4 \mathrm{~mm} ; \\
20-80 \% \text { infill; } \\
\mathrm{W}_{\mathrm{L}}=2\end{array}$ & $\begin{array}{c}\text { Bending- } \\
\text { ASTM D790; } \\
\text { Tensile-ASTM } \\
\text { D790; } \\
\mathrm{s}_{\mathrm{t}}=1 \mathrm{~mm} / \mathrm{min} \\
\end{array}$ & $\begin{array}{l}\text { Low variation of the } \\
\text { flexural strength and the } \\
\text { tensile strength } \\
\text { with } \alpha_{X Y} \text {. }\end{array}$ \\
\hline [58] & $\begin{array}{l}\alpha_{X Y}= \\
0^{\circ}-45^{\circ}\end{array}$ & $0.125-0.25$ & - & - & - & - & $50-90 \%$ infill & $\begin{array}{c}\text { Tensile-ISO } \\
527-1,2 \\
\end{array}$ & $\begin{array}{l}\text { Low variation of UTS vs. } \\
\text { the } \alpha_{X Y} \text { angle. }\end{array}$ \\
\hline [59] & $\begin{array}{l}\alpha_{X Y}= \\
0^{\circ}-90^{\circ}\end{array}$ & $0.10-0.35$ & $40-80$ & 220 & 25 & - & $\begin{array}{c}\mathrm{d}_{\mathrm{f}}=1.75 \mathrm{~mm} \\
100 \% \text { infill }\end{array}$ & $\begin{array}{c}\text { Tensile-ASTM } \\
\text { D638, Type V } \\
\text { specimens }\end{array}$ & $\begin{array}{c}\text { Higher E and UTS for } \\
\alpha_{X Y}=0^{\circ} \text { and } \alpha_{X Y}=45^{\circ} .\end{array}$ \\
\hline [64] & $\begin{array}{l}\gamma \mathrm{XY}= \\
0^{\circ}-90^{\circ}\end{array}$ & $0.10-0.30$ & - & 210 & 80 & $\begin{array}{l}30^{\circ} ; 45^{\circ} \\
60^{\circ}\end{array}$ & $\begin{array}{c}\mathrm{d}_{\mathrm{f}}=1.75 \mathrm{~mm} \\
50 \% \text { infill }\end{array}$ & $\begin{array}{l}\text { Tensile-ASTM } \\
\text { D638 }\end{array}$ & $\begin{array}{c}\text { Highest UTS for } \\
\gamma \mathrm{XY}=0^{\circ} \text { and } \gamma \mathrm{XY}=45^{\circ} \\
\text { specimens. }\end{array}$ \\
\hline [74] & $\begin{array}{l}\beta_{\mathrm{YZ}}= \\
0^{\circ}-90^{\circ}\end{array}$ & $0.05-0.20$ & 60 & 195-230 & 60 & - & $\begin{array}{c}\mathrm{d}_{\mathrm{f}}=1.75 \mathrm{~mm} \\
\mathrm{~d}_{\mathrm{n}}=0.4 \mathrm{~mm}\end{array}$ & $\begin{array}{c}\text { Tensile-ISO } \\
527-2 ; \\
\mathrm{s}_{\mathrm{t}}=2 \mathrm{~mm} / \mathrm{min} \\
\end{array}$ & $\begin{array}{c}\text { High decrease in UTS } \\
\text { with the increase in } \beta_{Y Z} \text {. }\end{array}$ \\
\hline
\end{tabular}


Table 5. Cont.

\begin{tabular}{|c|c|c|c|c|c|c|c|c|c|}
\hline \multirow[b]{2}{*}{ Ref. } & \multicolumn{7}{|c|}{ FFF Process Parameters } & \multirow{2}{*}{$\begin{array}{l}\text { Mechanical } \\
\text { Test Settings }\end{array}$} & \multirow{2}{*}{$\begin{array}{l}\text { Results and } \\
\text { Conclusions }\end{array}$} \\
\hline & $\begin{array}{c}\text { B.O. } \\
(-)\end{array}$ & $\begin{array}{c}t \\
(\mathrm{~mm})\end{array}$ & $\begin{array}{c}\mathrm{s}_{\mathrm{p}} \\
(\mathrm{mm} / \mathrm{s})\end{array}$ & $\begin{array}{c}\mathrm{T}_{\mathrm{H}} \\
\left({ }^{\circ} \mathrm{C}\right)\end{array}$ & $\begin{array}{c}\mathrm{T}_{\mathrm{B}} \\
\left({ }^{\circ} \mathrm{C}\right)\end{array}$ & $\begin{array}{c}\theta \\
\left({ }^{\circ}\right)\end{array}$ & $\begin{array}{c}\text { Other } \\
\text { Parameters }\end{array}$ & & \\
\hline [76] & $\begin{array}{l}\gamma \times z= \\
0^{\circ}-90^{\circ}\end{array}$ & $0.10-0.30$ & - & 215 & - & - & $\mathrm{d}_{\mathrm{f}}=1.75 \mathrm{~mm}$ & $\begin{array}{c}\text { Tensile-ISO } \\
527-2\end{array}$ & $\begin{array}{c}\text { High variation of UTS } \\
\text { with the } \gamma \mathrm{xZ} \text { angle, from } \\
55.86 \mathrm{MPa}(\mathrm{XZ} \\
\left.\text { specimens, } \gamma_{\mathrm{XZ}}=0^{\circ}\right) \text { to } \\
26.65 \mathrm{MPa}(\mathrm{ZX} \\
\left.\text { specimens, } \gamma_{\mathrm{XZ}}=90^{\circ}\right) .\end{array}$ \\
\hline [78] & $\begin{array}{c}\gamma \times \mathrm{xz}= \\
0^{\circ}-90^{\circ}\end{array}$ & $0.10-0.60$ & - & - & - & - & $\begin{array}{c}\mathrm{d}_{\mathrm{f}}=1.75 \mathrm{~mm} \\
\mathrm{~d}_{\mathrm{n}}=0.4 \mathrm{~mm}\end{array}$ & $\begin{array}{c}\text { Tensile-ISO } \\
527-2 ; \\
\mathrm{s}_{\mathrm{t}}=0.1 \\
\mathrm{~mm} / \mathrm{min}\end{array}$ & $\begin{array}{c}\text { High variation of UTS } \\
\text { with the } \gamma \mathrm{xZ} \text { angle, from } \\
51.33 \mathrm{MPa}(\mathrm{XZ} \\
\left.\text { specimens, } \gamma_{\mathrm{XZ}}=0^{\circ}\right) \text { to } \\
34.56 \mathrm{MPa}(\mathrm{ZX} \\
\left.\text { specimens, } \gamma_{\mathrm{XZ}}=90^{\circ}\right) .\end{array}$ \\
\hline [79] & $\begin{array}{l}\gamma \times z= \\
0^{\circ}-90^{\circ}\end{array}$ & $0.10-0.30$ & - & 220 & 60 & - & $\mathrm{d}_{\mathrm{f}}=1.75 \mathrm{~mm}$ & $\begin{array}{c}\text { Tensile-ISO } \\
527-2\end{array}$ & $\begin{array}{c}\text { High variation of UTS } \\
\text { with the } \gamma_{\mathrm{XZ}} \text { angle, from } \\
49.66 \mathrm{MPa}(\mathrm{XZ} \\
\left.\text { specimens, } \gamma_{\mathrm{XZ}}=0^{\circ}\right) \text { to } \\
23.40 \mathrm{MPa}(Z \mathrm{ZX} \\
\left.\text { specimens, } \gamma_{\mathrm{XZ}}=90^{\circ}\right) .\end{array}$ \\
\hline [101] & $\begin{array}{c}\alpha_{\mathrm{XY}}= \\
0^{\circ}-90^{\circ} \\
\beta_{\mathrm{YZ}}= \\
0^{\circ}-90^{\circ} \\
\gamma \mathrm{XZ}= \\
0^{\circ}-90^{\circ}\end{array}$ & 0.10 & - & - & - & - & $\begin{array}{c}\mathrm{d}_{\mathrm{n}}=0.4 \mathrm{~mm} \\
99 \% \text { infill }\end{array}$ & $\begin{array}{c}\text { Tensile-ISO } \\
527-2\end{array}$ & $\begin{array}{c}\text { Highest UTS }(55.68 \\
\text { MPa) for XZ }\left(\gamma \times Z=0^{\circ}\right) \text {; } \\
\text { Low UTS }(12.68-15.5 \\
\text { MPa) for YX, YZ, } \\
\beta_{Y Z}=45^{\circ} \text { and } \\
\alpha_{X Y}=45^{\circ} \text { specimens. }\end{array}$ \\
\hline [102] & $\begin{array}{l}\gamma \times Y= \\
0^{\circ}-90^{\circ} \\
\gamma \times Z= \\
0^{\circ}-90^{\circ}\end{array}$ & 0.2 & 50 & 225 & 60 & - & $\begin{array}{c}\mathrm{d}_{\mathrm{f}}=2.75 \mathrm{~mm} \\
\mathrm{~d}_{\mathrm{n}}=0.6 \mathrm{~mm}\end{array}$ & $\begin{array}{c}\text { Tensile-ISO } \\
527 ; \\
\text { Bending-ISO } \\
\text { 178; } \\
\text { Compression- } \\
\text { ISO } 604\end{array}$ & $\begin{array}{l}\text { Highest UTS }(49.8 \mathrm{MPa}) \\
\text { for XZ }\left(\gamma_{X Z}=0^{\circ}\right) . \\
\text { Lowest UTS }(21.5 \mathrm{MPa}) \\
\text { for } Z Y \text { and } Z \mathrm{XX} \text {. UTS } \\
\text { decreases with the } \\
\text { increase in } \gamma_{\mathrm{XY}} \text { and } \gamma_{\mathrm{XZ}} \text {. } \\
\text { Low variation of the } \\
\text { compressive strength. }\end{array}$ \\
\hline [103] & $\begin{array}{l}\gamma \mathrm{XY}= \\
0^{\circ}-90^{\circ} \\
\gamma_{X Z}= \\
0^{\circ}-90^{\circ}\end{array}$ & 0.15 & 60 & 220 & 60 & - & $\begin{array}{c}\mathrm{d}_{\mathrm{f}}=1.75 \mathrm{~mm} ; \\
\mathrm{d}_{\mathrm{n}}=0.4 \mathrm{~mm} \\
25-100 \% \text { infill }\end{array}$ & $\begin{array}{c}\text { Tensile-ASTM } \\
\text { D638; } \\
\text { Shear-ASTM } \\
\text { D5379 }\end{array}$ & $\begin{array}{c}\text { High decrease in UTS } \\
\text { with the increase in } \gamma \mathrm{XZ} \text {. } \\
\mathrm{UTS}=55 \mathrm{MPa} \text { for XZ } \\
\left(\gamma_{\mathrm{XZ}}=0^{\circ}\right) \text {. Highest } \\
\text { shear strength }(36 \mathrm{MPa}) \\
\text { for } \gamma_{\mathrm{XY}}=45^{\circ} .\end{array}$ \\
\hline [104] & $\begin{array}{c}\beta_{Y X}= \\
0^{\circ}-90^{\circ} ; \\
\beta_{X Y}= \\
0^{\circ}-90^{\circ} ; \\
\beta_{Y Z}= \\
0^{\circ}-90^{\circ} \\
\end{array}$ & 0.20 & 35 & 205 & 60 & $\begin{array}{l}0^{\circ} / 90^{\circ} ; \\
30^{\circ} /-60^{\circ} ; \\
45^{\circ} /-45^{\circ} ; \\
60^{\circ} /-30^{\circ} \\
90^{\circ} / 0^{\circ}\end{array}$ & $\begin{array}{c}\mathrm{d}_{\mathrm{f}}=1.75 \mathrm{~mm} \\
\mathrm{~d}_{\mathrm{n}}=0.4 \mathrm{~mm} \\
10 \% \text { infill }\end{array}$ & $\begin{array}{c}\text { Tensile-ASTM } \\
\text { D638 }\end{array}$ & $\begin{array}{l}\text { Low influence of } \beta_{X Y} \text {. } \\
\text { High influence of } \beta_{Y X} \\
\text { and } \beta_{Y Z} \text {. Highest UTS } \\
(27.6 \mathrm{MPa}-30.9 \mathrm{MPa}) \text { for } \\
\beta_{X Y}=0^{\circ}-90^{\circ}, \beta_{Y X}=0^{\circ} \\
\text { and } \beta_{Y Z}=0^{\circ} \text { specimens. }\end{array}$ \\
\hline
\end{tabular}

Analyzing the data presented in Table 5, it can be concluded that the ZX- and ZY-type build orientations lead to much lower mechanical characteristics compared to the $X Y, Y X$, $\mathrm{XZ}$ and $\mathrm{YZ}$ layouts. This mechanical behavior is generated by the inter-layer breakage that occurs in $\mathrm{ZX}$ and $\mathrm{ZY}$ specimens.

At tilted specimens relative to the build plate $\left(0^{\circ}<\beta_{Y Z}<90^{\circ} ; 0^{\circ}<\beta_{Y X}<90^{\circ}\right.$; $\left.0^{\circ}<\gamma_{\mathrm{XZ}}<90^{\circ} ; 0^{\circ}<\gamma_{\mathrm{XY}}<90^{\circ}\right)$, the mechanical characteristics decrease with increases in the tilt angle.

A comparative analysis of the $\mathrm{XY}$ and the $\mathrm{YX}$ build orientations should be correlated with the raster angle (similar for specimens with $0^{\circ}<\alpha_{X Y}<90^{\circ}$ ).

The anisotropic character of components made by FFF printing was also evidenced by some authors through mechanical tests performed on specimens obtained by conventional machining (cutting) from 3D-printed prismatic blocks [105].

\section{Raster Angle}

The raster of the 3D-printed parts represents the arrangement of the successive lines of a layer (Figure 3). The mechanical behavior is influenced by several raster parameters: the raster angle, how the raster angle alternates between two successive layers, the width of a raster line, the distance between two successive raster lines, the number of wall lines and the distance between the raster and the wall lines [106]. 


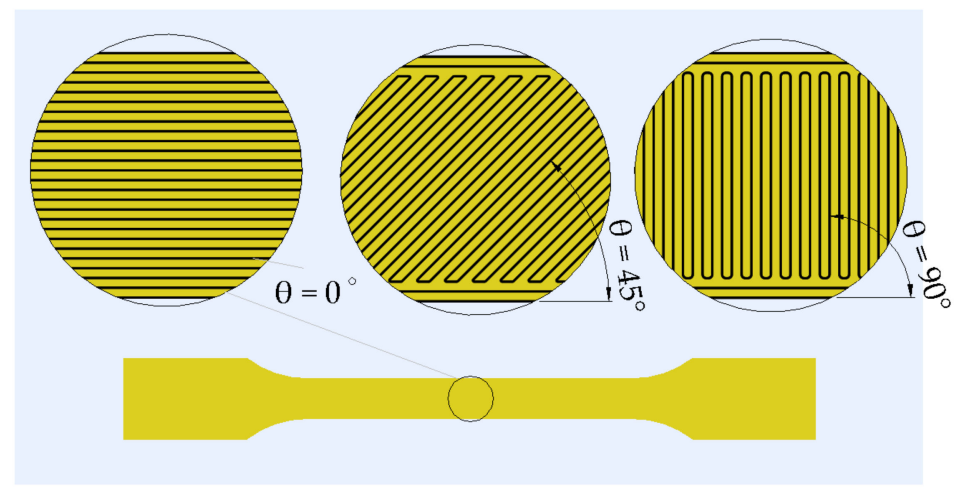

Figure 3. Raster angle $(\theta)$.

The raster angle influences the anisotropic mechanical behavior and the breakage of 3D FFF-printed components. Two main types of layouts are distinguished: unidirectional raster (the same raster angle is maintained for all successive layers) and alternating raster (the raster angle varies between successive layers, usually by $90^{\circ}$ ). Even in the case of the raster angle, a standardization of notations is needed with a clear identification of the alternating raster. Therefore, notations in the form of $\theta_{1}^{\circ} / \theta_{2}^{\circ}$ could be used, where $\theta_{1}^{\circ}$ and $\theta_{2}{ }^{\circ}$ represent the raster angles for two successive layers.

In the previous research were analyzed specimens with unidirectional raster and alternating raster (Table 6-the first 11 lines of the table show unidirectional raster, the next 14 lines of the table show alternating raster). The highest mechanical properties were obtained for the specimens with alternating raster. The analysis of the mechanical behavior as a function of the angle of the raster should be carried out correlated with the specimen build orientation.

Table 6. The influence of the raster angle on the mechanical properties of FFF-printed PLA.

\begin{tabular}{|c|c|c|c|c|c|c|c|c|c|}
\hline \multirow[b]{2}{*}{ Ref. } & \multicolumn{7}{|c|}{ FFF Process Parameters } & \multirow{2}{*}{$\begin{array}{l}\text { Mechanical Test } \\
\text { Settings }\end{array}$} & \multirow[b]{2}{*}{$\begin{array}{l}\text { Results and } \\
\text { Conclusions }\end{array}$} \\
\hline & $\begin{array}{c}\theta \\
\left({ }^{\circ}\right)\end{array}$ & $\begin{array}{c}\mathrm{t} \\
(\mathrm{mm})\end{array}$ & $\begin{array}{c}\mathrm{s}_{\mathrm{p}} \\
(\mathrm{mm} / \mathrm{s})\end{array}$ & $\begin{array}{l}\mathrm{T}_{\mathrm{H}} \\
\left({ }^{\circ} \mathrm{C}\right)\end{array}$ & $\begin{array}{l}\mathrm{T}_{\mathrm{B}} \\
\left({ }^{\circ} \mathrm{C}\right)\end{array}$ & $\begin{array}{l}\text { B.O. } \\
(-)\end{array}$ & $\begin{array}{c}\text { Other } \\
\text { Parameters }\end{array}$ & & \\
\hline \multicolumn{10}{|c|}{$\begin{array}{c}\theta \text {-raster angle; } t \text { - layer thickness (layer height); } \mathrm{s}_{\mathrm{p}} \text {-printing speed; } \mathrm{T}_{\mathrm{H}} \text {-printing head (nozzle) temperature; } \mathrm{T}_{\mathrm{B}}-\text { build plate temperature; } \\
\text { B.O.- - build orientation; } \mathrm{d}_{\mathrm{f}} \text {-filament diameter; } \mathrm{d}_{\mathrm{n}} \text {-nozzle diameter; } \mathrm{W}_{\mathrm{L}} \text {-number of wall lines. }\end{array}$} \\
\hline [24] & $40^{\circ} ; 60^{\circ} ; 80^{\circ}$ & $0.10-0.30$ & 30 & 195 & 110 & Horizontal & $\begin{array}{l}\mathrm{d}_{\mathrm{f}}=1.75 \mathrm{~mm} ; \\
\mathrm{d}_{\mathrm{n}}=0.3 \mathrm{~mm} ; \\
20-80 \% \text { infill }\end{array}$ & $\begin{array}{c}\text { Tensile-ASTM } \\
\text { D638 }\end{array}$ & $\begin{array}{c}\text { The variation of UTS vs. } \\
\theta \text { is influenced by the } \\
\text { layer thickness. }\end{array}$ \\
\hline [27] & $0^{\circ} ; 45^{\circ} ; 90^{\circ}$ & 0.20 & - & - & - & $\mathrm{XY} ; \mathrm{XZ} ; \mathrm{ZX}$ & $50-100 \%$ infill & $\begin{array}{c}\text { Tensile-ISO } \\
527-2 ; \\
\mathrm{s}_{\mathrm{t}}=10 \mathrm{~mm} / \mathrm{min}\end{array}$ & $\begin{array}{l}\text { A decrease of } 16.7 \% \text { of } \\
\text { the UTS for } \theta=90^{\circ} \\
\text { compared to } \theta=0^{\circ} \text { and } \\
\theta=45^{\circ} \text { specimens. }\end{array}$ \\
\hline [29] & $\begin{array}{c}0^{\circ} ; 30^{\circ} ; 45^{\circ} \\
60^{\circ} ; 90^{\circ}\end{array}$ & 0.40 & $20-80$ & 215 & 55 & Horizontal & $\begin{array}{c}100 \% \text { infill; } \\
\mathrm{W}_{\mathrm{L}}=2\end{array}$ & $\begin{array}{l}\text { Tensile_ASTM } \\
\text { D638; } \\
\mathrm{S}_{\mathrm{t}}=5 \mathrm{~mm} / \mathrm{min}\end{array}$ & $\begin{array}{c}\text { For } \mathrm{t}=0.40 \mathrm{~mm} \text { all } \\
\text { specimens fractured in } \\
\text { the direction of the } \\
\text { raster. Highest UTS for } \\
\theta=0^{\circ} \text { specimens; UTS } \\
\text { decreases by approx. } \\
40 \% \text { for } \theta=90^{\circ} \\
\text { specimens. }\end{array}$ \\
\hline [37] & $0^{\circ} ; 45^{\circ} ; 90^{\circ}$ & 0.10 & 30 & 240 & 60 & Horizontal & $\begin{array}{c}\mathrm{d}_{\mathrm{f}}=2.85 \mathrm{~mm} ; \\
\mathrm{d}_{\mathrm{n}}=0.4 \mathrm{~mm} ; \\
100 \% \text { infill; } \\
1-10 \% \text { moisture }\end{array}$ & $\begin{array}{c}\text { Tensile-ASTM } \\
\text { D638; } \\
\mathrm{s}_{\mathrm{t}}=5 \mathrm{~mm} / \mathrm{min}\end{array}$ & $\begin{array}{l}\text { The raster angle has a } \\
\text { high significance on } \\
\text { UTS. Maximum UTS } \\
(50.3 \mathrm{MPa}) \text { and E } \\
(1890 \mathrm{MPa}) \text { obtained at } \\
\begin{array}{c}\theta=0^{\circ} \text { and } 10 \% \\
\text { moisture content. }\end{array}\end{array}$ \\
\hline [61] & $0^{\circ} ; 90^{\circ}$ & $0.20-0.30$ & $38-52$ & 190 & 40 & - & $\begin{array}{c}\mathrm{d}_{\mathrm{n}}=0.40 \mathrm{~mm} \\
40 \% \text { infill }\end{array}$ & $\begin{array}{c}\text { Bending- } \\
\text { ASTM D790; } \\
\mathrm{s}_{\mathrm{t}}=12 \mathrm{~mm} / \mathrm{min}\end{array}$ & $\begin{array}{l}\text { A higher flexural } \\
\text { strength for } \\
\theta=0^{\circ} \text { specimens. }\end{array}$ \\
\hline [63] & $\begin{array}{c}0^{\circ} ; 18^{\circ} ; 45^{\circ} ; \\
72^{\circ} ; 90^{\circ}\end{array}$ & $0.10-0.20$ & 60 & 205 & 60 & Horizontal & $\begin{array}{l}100 \% \text { infill; } \\
W_{L}=2-6\end{array}$ & $\begin{array}{l}\text { Tensile-ASTM } \\
\text { D638 modified } \\
\text { specimens }\end{array}$ & $\begin{array}{c}\text { Highest UTS } \\
(53.59 \mathrm{MPa}) \text { and } \mathrm{E} \\
(3388.57 \mathrm{MPa}) \text { for } \theta=0^{\circ} \\
\text { Lowest UTS }(43.39 \mathrm{MPa}) \\
\text { and } \mathrm{E}(2799.43 \mathrm{MPa}) \text { for } \\
\theta=90^{\circ} .\end{array}$ \\
\hline
\end{tabular}


Table 6. Cont.

\begin{tabular}{|c|c|c|c|c|c|c|c|c|c|}
\hline \multirow[b]{2}{*}{ Ref. } & \multicolumn{7}{|c|}{ FFF Process Parameters } & \multirow{2}{*}{$\begin{array}{l}\text { Mechanical Test } \\
\text { Settings }\end{array}$} & \multirow{2}{*}{$\begin{array}{l}\text { Results and } \\
\text { Conclusions }\end{array}$} \\
\hline & $\begin{array}{c}\theta \\
\left({ }^{\circ}\right)\end{array}$ & $\begin{array}{c}\mathrm{t} \\
(\mathrm{mm})\end{array}$ & $\begin{array}{c}\mathrm{s}_{\mathrm{p}} \\
(\mathrm{mm} / \mathrm{s})\end{array}$ & $\begin{array}{c}\mathrm{T}_{\mathrm{H}} \\
\left({ }^{\circ} \mathrm{C}\right)\end{array}$ & $\begin{array}{c}\mathrm{T}_{\mathrm{B}} \\
\left({ }^{\circ} \mathrm{C}\right)\end{array}$ & $\begin{array}{c}\text { B.O. } \\
(-)\end{array}$ & $\begin{array}{c}\text { Other } \\
\text { Parameters }\end{array}$ & & \\
\hline [64] & $30^{\circ} ; 45^{\circ} ; 60^{\circ}$ & $0.10-0.30$ & - & 210 & 80 & $\begin{array}{l}\gamma_{X Y}= \\
0^{\circ}-90^{\circ}\end{array}$ & $\begin{array}{c}\mathrm{d}_{\mathrm{f}}=1.75 \mathrm{~mm} ; \\
50 \% \text { infill }\end{array}$ & $\begin{array}{c}\text { Tensile_ASTM } \\
\text { D638 }\end{array}$ & $\begin{array}{l}\text { UTS decreases with the } \\
\text { increase in } \theta \text {. }\end{array}$ \\
\hline [69-71] & $0^{\circ} ; 45^{\circ} ; 90^{\circ}$ & $0.10-0.30$ & 50 & 210 & 70 & - & $\begin{array}{c}\mathrm{d}_{\mathrm{f}}=1.75 \mathrm{~mm} \\
\mathrm{~d}_{\mathrm{n}}=0.4 \mathrm{~mm} \\
100 \% \text { infill; } \\
\mathrm{W}_{\mathrm{L}}=1\end{array}$ & $\begin{array}{l}\text { Tensile-ASTM } \\
\text { D638; } \\
\text { Bending- } \\
\text { ASTM D790; } \\
\text { Impact_ASTM } \\
\text { D256 }\end{array}$ & $\begin{array}{l}\text { High influence of the } \\
\text { raster angle on the } \\
\text { mechanical properties. } \\
\text { Highest UTS, UFS and } \\
\text { Izod impact strength for } \\
\theta=0^{\circ} \text { specimens. }\end{array}$ \\
\hline$[90]$ & $\begin{array}{c}0^{\circ} ; 45^{\circ} \\
90^{\circ}\end{array}$ & 0.35 & 40 & $190-210$ & 55 & Horizontal & $\begin{array}{c}\mathrm{d}_{\mathrm{f}}=2.85 \mathrm{~mm} ; \\
\mathrm{d}_{\mathrm{n}}=0.4 \mathrm{~mm} \\
\mathrm{~W}_{\mathrm{L}}=2\end{array}$ & $\begin{array}{l}\text { Tensile-ASTM } \\
\quad \text { D638-10-I; } \\
\mathrm{s}_{\mathrm{t}}=5 \mathrm{~mm} / \mathrm{min}\end{array}$ & $\begin{array}{l}\text { Highest UTS and E for } \\
\begin{array}{l}\theta=0^{\circ} \text { specimens. } \\
\text { Lowest UTS and E for } \\
\theta=90^{\circ} \text { specimens. }\end{array}\end{array}$ \\
\hline [107] & $\begin{array}{c}0^{\circ} ; 30^{\circ} ; 45^{\circ} \\
60^{\circ} ; 90^{\circ}\end{array}$ & 0.20 & 30 & 200 & 60 & Horizontal & $100 \%$ infill & $\begin{array}{l}\text { Tensile-ISO } \\
\text { 527-2, Type 1B } \\
\text { specimens }\end{array}$ & $\begin{array}{l}\text { Breaking surface aligned } \\
\text { with the raster. } \\
\text { Highest UTS and E for } \\
\theta=0^{\circ} \text {; UTS decreases by } \\
\text { approx. } 70 \% \text { for } \theta=90^{\circ} \text {. }\end{array}$ \\
\hline [108] & $\begin{array}{c}0^{\circ} ; 30^{\circ} ; 45^{\circ} \\
60^{\circ} ; 90^{\circ}\end{array}$ & - & 70 & 200 & 60 & $X Y$ & $\begin{array}{c}\mathrm{d}_{\mathrm{f}}=2.85 \mathrm{~mm} \\
100 \% \text { infill }\end{array}$ & $\begin{array}{c}\text { Tensile_ASTM } \\
\text { D638 }\end{array}$ & $\begin{array}{l}\text { High influence of the } \\
\text { raster angle on UTS. } \\
\text { Highest UTS for } \theta=45^{\circ} \text {. }\end{array}$ \\
\hline [27] & $\begin{array}{c}0^{\circ} / 45^{\circ} ; \\
45^{\circ} /-45^{\circ} \\
0^{\circ} / 90^{\circ}\end{array}$ & 0.20 & - & - & - & $X Y ; X Z ; Z X$ & $50-100 \%$ infill & $\begin{array}{c}\text { Tensile-ISO } \\
527-2 ; \\
\mathrm{s}_{\mathrm{t}}=10 \mathrm{~mm} / \mathrm{min} \\
\end{array}$ & $\begin{array}{l}\text { Highest UTS (58.4 MPa) } \\
\text { for } \theta=0^{\circ} / 45^{\circ} \text {. }\end{array}$ \\
\hline [32] & $\begin{array}{c}45^{\circ} /-45^{\circ} ; \\
0^{\circ} / 90^{\circ} ; \\
0^{\circ} /-30^{\circ} / 30^{\circ} / \\
-60^{\circ} / 60^{\circ} / 90^{\circ}\end{array}$ & $0.10-0.30$ & 60 & 215 & 60 & Horizontal & $\begin{array}{c}\mathrm{d}_{\mathrm{f}}=1.75 \mathrm{~mm} ; \\
100 \% \text { infill; } \\
\text { ageing; } \\
\text { heat treatment }\end{array}$ & $\begin{array}{c}\text { Tensile_ASTM } \\
\text { D638 }\end{array}$ & $\begin{array}{c}\text { Higher UTS for } \\
\theta=-45^{\circ} / 45^{\circ} \text {. The } \\
\text { variation of UTS vs. } \\
\text { raster angle is } \\
\text { influenced by heat } \\
\text { treatment and ageing. }\end{array}$ \\
\hline [33] & $\begin{array}{l}0^{\circ} / 90^{\circ} \\
15^{\circ} / 75^{\circ} \\
30 / 60^{\circ} \\
45 / 45^{\circ}\end{array}$ & - & 50 & $190-230$ & $45-105$ & - & $\begin{array}{c}\mathrm{d}_{\mathrm{f}}=2.85 \mathrm{~mm} \\
100 \% \text { infill }\end{array}$ & $\begin{array}{l}\text { Tensile-ASTM } \\
\text { D638; } \\
\mathrm{s}_{\mathrm{t}}=5 \mathrm{~mm} / \mathrm{min} \\
\text { Bending- } \\
\text { ASTM D790; } \\
\text { Impact-ASTM } \\
\text { D256 } \\
\end{array}$ & $\begin{array}{l}\text { Highest values of tensile } \\
\text { strength, flexural } \\
\text { strength and Izod } \\
\text { impact strength } \\
\text { obtained for } \theta=45 / 45^{\circ} \text {. }\end{array}$ \\
\hline [51] & $\begin{array}{c}0^{\circ} ; 90^{\circ} ; \\
45^{\circ} /-45^{\circ}\end{array}$ & $0.06-0.35$ & 60 & $190-220$ & 60 & $X Y$ & $\begin{array}{c}\mathrm{d}_{\mathrm{f}}=1.75 \mathrm{~mm} ; \\
\mathrm{d}_{\mathrm{n}}=0.4 \mathrm{~mm} ; \\
100 \% \text { infill; } \\
\mathrm{W}_{\mathrm{L}}=2\end{array}$ & $\begin{array}{l}\text { Tensile-ASTM } \\
\text { D638-I } \\
\text { specimens; } \\
\mathrm{s}_{\mathrm{t}}=5 \mathrm{~mm} / \mathrm{min}\end{array}$ & $\begin{array}{c}\text { Highest UTS for } \\
\theta=45 /-45^{\circ} \text {. Low } \\
\text { values of UTS for } \\
\text { specimens with } \theta=90^{\circ} \\
\text { and } \mathrm{t}=0.06 \mathrm{~mm} \text {. }\end{array}$ \\
\hline [83] & $\begin{array}{c}0^{\circ} / 90^{\circ} \\
30^{\circ} /-60^{\circ} \\
45^{\circ} /-45^{\circ}\end{array}$ & $0.08-0.28$ & $20-60$ & $210-220$ & - & $X Y ; X Z$ & $\begin{array}{c}\mathrm{d}_{\mathrm{f}}=1.75 \mathrm{~mm} ; \\
\mathrm{d}_{\mathrm{n}}=0.3-0.5 \mathrm{~mm} ; \\
80-100 \% \text { infill; } \\
\mathrm{W}_{\mathrm{L}}=2-4\end{array}$ & $\begin{array}{c}\text { Tensile test, } \\
\text { ASTM D638-I; } \\
\mathrm{s}_{\mathrm{t}}=5 \mathrm{~mm} / \mathrm{min}\end{array}$ & $\begin{array}{c}\text { Higher UTS for } \\
\text { specimens with } \\
\begin{array}{c}\theta=30^{\circ} /-60^{\circ} \text { and } \\
\theta=45^{\circ} /-45^{\circ}\end{array}\end{array}$ \\
\hline [86] & $\begin{array}{c}45^{\circ} /-45^{\circ} \\
0^{\circ} / 90^{\circ}\end{array}$ & 0.10 & $35-65$ & 200 & 60 & $X Y$ & $\begin{array}{c}\mathrm{d}_{\mathrm{f}}=2.85 \mathrm{~mm} ; \\
100 \% \text { infill }\end{array}$ & $\begin{array}{c}\text { Tensile_ASTM } \\
\text { D638 } \\
\end{array}$ & $\begin{array}{l}\text { Higher UTS for } \\
\theta=45^{\circ} /-45^{\circ} .\end{array}$ \\
\hline [104] & $\begin{array}{l}0^{\circ} / 90^{\circ} ; \\
30^{\circ} /-60^{\circ} ; \\
45^{\circ} /-45^{\circ} ; \\
60^{\circ} /-30^{\circ} \\
90^{\circ} / 0^{\circ}\end{array}$ & 0.20 & 35 & 205 & 60 & $\begin{array}{c}\beta_{Y X}= \\
0^{\circ}-90^{\circ} ; \\
\beta_{X Y}= \\
0^{\circ}-90^{\circ} ; \\
\beta_{Y Z}= \\
0^{\circ}-90^{\circ}\end{array}$ & $\begin{array}{c}\mathrm{d}_{\mathrm{f}}=1.75 \mathrm{~mm} \\
\mathrm{~d}_{\mathrm{n}}=0.4 \mathrm{~mm} \\
10 \% \text { infill }\end{array}$ & $\begin{array}{c}\text { Tensile_ASTM } \\
\text { D638 }\end{array}$ & $\begin{array}{l}\text { Low influence }(2 \mathrm{MPa}) \\
\text { of the raster angle on } \\
\text { UTS, at } 10 \% \text { infill. }\end{array}$ \\
\hline [107] & $\begin{array}{l}0^{\circ} / 90^{\circ} \\
30^{\circ} /-60^{\circ} \\
45^{\circ} /-45^{\circ}\end{array}$ & 0.20 & 30 & 200 & 60 & Horizontal & $100 \%$ infill & $\begin{array}{l}\text { Tensile-ISO } \\
\text { 527-2, Type 1B } \\
\text { specimens }\end{array}$ & $\begin{array}{l}\text { Low influence of the } \\
\text { alternating raster angle } \\
\text { on the elastic modulus } \\
\text { and the ultimate } \\
\text { tensile strength. }\end{array}$ \\
\hline [109] & $\begin{array}{l}0^{\circ} / 90^{\circ} \\
15^{\circ} /-75^{\circ} \\
30^{\circ} /-60^{\circ} \\
45^{\circ} /-45^{\circ}\end{array}$ & 0.20 & 55 & 210 & 60 & Horizontal & - & $\begin{array}{c}\text { Tensile-ASTM } \\
\text { D638; } \\
\mathrm{s}_{\mathrm{t}}=0.5 \\
\mathrm{~mm} / \mathrm{min} ; \\
\text { fracture test }\end{array}$ & $\begin{array}{c}\text { Highest } \mathrm{E}(1942 \mathrm{MPa}) \\
\text { and yield stress } \\
(27.1 \mathrm{MPa}) \text { for } \theta=0 / 90^{\circ} ; \\
\text { Highest fracture load } \\
(865.1 \mathrm{~N}) \text { in fracture test } \\
\text { of specimens with } \\
\theta=45 /-45^{\circ} .\end{array}$ \\
\hline [110] & $\begin{array}{r}0^{\circ} ; 90^{\circ} \\
45 /-45^{\circ}\end{array}$ & 0.30 & 50 & 190 & 55 & $X Y$ & $\mathrm{~d}_{\mathrm{n}}=0.4 \mathrm{~mm}$ & $\begin{array}{l}\text { Tensile_ASTM } \\
\text { D638; } \\
\mathrm{s}_{\mathrm{t}}=5 \mathrm{~mm} / \mathrm{min}\end{array}$ & $\begin{array}{l}\text { Highest UTS for } \theta=0^{\circ} ; \\
\text { Low influence of the } \\
\text { raster angle on the } \\
\text { elastic modulus for PLA. }\end{array}$ \\
\hline [111] & $\begin{array}{c}0^{\circ} / 90^{\circ} \\
45^{\circ} /-45^{\circ}\end{array}$ & 0.2 & 120 & 200 & 50 & Horizontal & $\begin{array}{c}\mathrm{d}_{\mathrm{f}}=1.75 \mathrm{~mm} \\
\mathrm{~d}_{\mathrm{n}}=0.4 \mathrm{~mm} ; \\
3090 \% \text { infill; } \\
\mathrm{W}_{\mathrm{L}}=2\end{array}$ & $\begin{array}{l}\text { Tensile-ISO } \\
527\end{array}$ & $\begin{array}{c}\text { A higher strength of } \\
\text { specimens for } \\
\theta=45^{\circ} /-45^{\circ} \text {. Low } \\
\text { influence of raster angle } \\
\text { on elastic modulus. }\end{array}$ \\
\hline
\end{tabular}


Table 6. Cont.

\begin{tabular}{|c|c|c|c|c|c|c|c|c|c|}
\hline \multirow[b]{2}{*}{ Ref. } & \multicolumn{7}{|c|}{ FFF Process Parameters } & \multirow{2}{*}{$\begin{array}{l}\text { Mechanical Test } \\
\text { Settings }\end{array}$} & \multirow{2}{*}{$\begin{array}{l}\text { Results and } \\
\text { Conclusions }\end{array}$} \\
\hline & $\begin{array}{c}\theta \\
\left({ }^{\circ}\right)\end{array}$ & $\begin{array}{c}\mathrm{t} \\
(\mathrm{mm})\end{array}$ & $\begin{array}{c}\mathrm{s}_{\mathrm{p}} \\
(\mathrm{mm} / \mathrm{s})\end{array}$ & $\begin{array}{c}\mathrm{T}_{\mathrm{H}} \\
\left({ }^{\circ} \mathrm{C}\right)\end{array}$ & $\begin{array}{c}\mathrm{T}_{\mathrm{B}} \\
\left({ }^{\circ} \mathrm{C}\right)\end{array}$ & $\begin{array}{c}\text { B.O. } \\
(-)\end{array}$ & $\begin{array}{c}\text { Other } \\
\text { Parameters }\end{array}$ & & \\
\hline [112] & $\begin{array}{c}0^{\circ} ; 90^{\circ} ; \\
45^{\circ} / 0^{\circ} / 90^{\circ} / \\
135^{\circ}\end{array}$ & 0.14 & 40 & 215 & 60 & $X Y$ & $\begin{array}{c}\mathrm{d}_{\mathrm{f}}=1.75 \mathrm{~mm} ; \\
\mathrm{d}_{\mathrm{n}}=0.4 \mathrm{~mm} ; \\
100 \% \text { infill }\end{array}$ & $\begin{array}{c}\text { Tensile-ASTM } \\
\text { D638 }\end{array}$ & $\begin{array}{c}\text { Highest UTS }(57.7 \mathrm{MPa}) \\
\text { for } \theta=0^{\circ} ; \\
\text { Lowest UTS }(30.8 \mathrm{MPa}) \\
\text { for } \theta=90^{\circ} .\end{array}$ \\
\hline [113] & $\begin{array}{c}45^{\circ} /-45^{\circ} \\
0^{\circ} / 90^{\circ}\end{array}$ & 0.15 & 40 & 210 & 50 & - & $\begin{array}{c}\mathrm{d}_{\mathrm{f}}=1.75 \mathrm{~mm} ; \\
\mathrm{d}_{\mathrm{n}}=0.5 \mathrm{~mm} ; \\
100 \% \text { infill }\end{array}$ & $\begin{array}{c}\text { Bending- } \\
\text { ASTM D790; } \\
\text { Compression- } \\
\text { ASTM S695; } \\
\text { Impact } \\
\text { test-ASTM } \\
\text { D256 }\end{array}$ & $\begin{array}{l}\text { Higher UFS }(+14.31 \%) \\
\text { and impact strength } \\
(+41.20 \%) \text { for } \\
\theta=45^{\circ} /-45^{\circ} \text {. Low } \\
\text { influence of raster angle } \\
\text { on the } \\
\text { compressive strength. }\end{array}$ \\
\hline [114] & $\begin{array}{l}45^{\circ} /-45^{\circ} \\
0^{\circ} / 90^{\circ}\end{array}$ & 0.25 & 50 & 210 & 60 & $X Y$ & $\begin{array}{c}\mathrm{d}_{\mathrm{f}}=1.75 \mathrm{~mm} ; \\
\mathrm{d}_{\mathrm{n}}=0.4 \mathrm{~mm} \\
100 \% \text { infill }\end{array}$ & $\begin{array}{c}\text { Tensile-ASTM } \\
\text { D638; } \\
\text { Bending- } \\
\text { ASTM D790; } \\
\text { Impact-ASTM } \\
\text { D256 }\end{array}$ & $\begin{array}{l}\text { Higher UTS and Izod } \\
\text { impact strength for } \\
\theta=45^{\circ} /-45^{\circ} \text {; Higher } \\
\text { UFS for } \theta=0^{\circ} / 90^{\circ} \text {. }\end{array}$ \\
\hline
\end{tabular}

The failure of tensile specimens can be influenced by raster and build orientations. Three failure modes can be defined:

- Inter-layer failure, when the failure occurs at the interface between two adjacent layers [77] (ex. the breaking of tensile specimens with ZY or ZX orientations);

- Inter-line failure (the breaking surface aligned with the raster angle-Figure 4);

- In-layer failure or in-line failure (the breaking surface is not aligned with the raster angle or the interface between two adjacent layers).

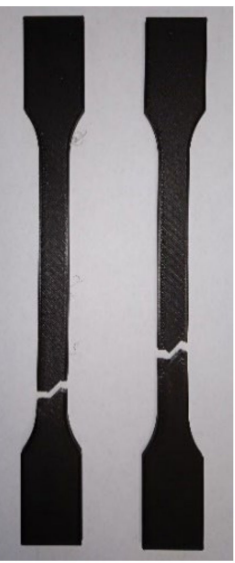

(a)

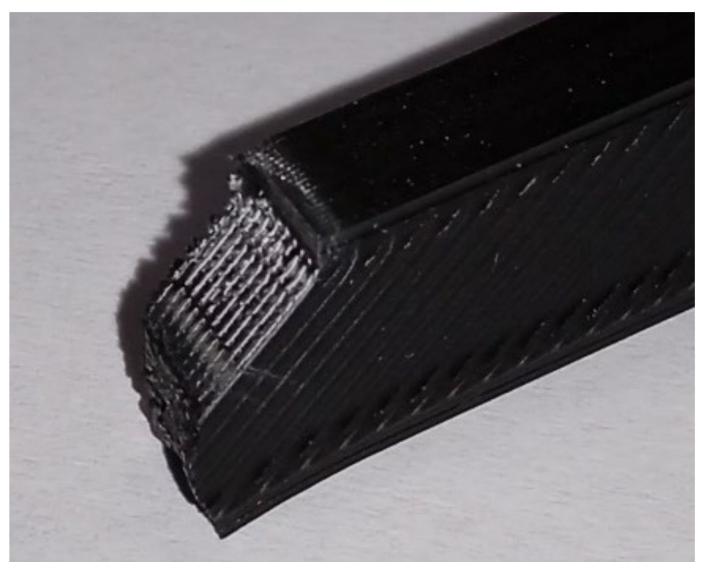

(b)

Figure 4. Breaking surface aligned with the raster angle; (a) ISO 527-2:2012 1A specimens; (b) detail.

Inter-line failure can be associated with reduced diffusion between the raster lines.

The occurrence of inter-line failure at the $X Y$ specimens with a unidirectional raster angle of $\theta=90^{\circ}$ is the cause for the lower tensile strength of these specimens relative to the tensile strength of specimens with $\theta=0^{\circ}$ or $\theta=45^{\circ}$.

The effect of stress concentration in the radius area of the tensile specimens may be amplified by the raster layout, mainly in the case of the unidirectional raster [1].

The optimization of the fused filament fabrication technology certainly has to start with the prioritization of the process parameters according to their impact on the mechanical properties of the printed part. In [22] a hierarchy of the influence of six process parameters on several mechanical properties is presented. For specimens printed in the $\mathrm{XZ}$ orientation, the order of the influence of the process parameters on the ultimate tensile strength is considered to be: infill density, layer thickness, presence of a contour wall, head temperature, infill orientation and printing speed, while in case of the $\mathrm{XY}$ orientation the 
order of the importance of these parameters is different: layer thickness, infill orientation, infill density, head temperature, printing speed and presence of a contour wall. This order changes when other mechanical parameters (Young's modulus, yield strength, etc.) are monitored.

\section{Discussions and Conclusions}

Fused filament fabrication is a widespread technology, used in various applications, ranging from industrial manufacturing and research activities to home use. Polylactic acid is a biodegradable, low-carbon-footprint material that can be used for the fabrication of industrial products if predictable and repeatable mechanical properties are achieved in the production process.

The mechanical behavior of components made of PLA by FFF is influenced by several factors along the production chain: filament manufacturing, geometrical design, process parameters, 3D-printing equipment, ageing and post-process treatments and mechanical testing procedure.

From the process parameters most investigated in the literature, in this paper the following have been analyzed: layer thickness, printing speed, printing head temperature, build plate temperature, build orientation and raster angle.

The necessity for standardization and uniformity in the definition of process parameters is highlighted. Comparative analysis of previous research is hampered by ambiguous or incomplete definitions of certain process parameters. Furthermore, the simultaneous variation of several process parameters during the experimental investigations conduces to difficulties in pointing out the influence of each parameter considered individually.

Finally, the critical need to define suitable specimens for the mechanical testing of FFF products is revealed by the large number of tensile specimens with breakage occurring outside the gauge length. Without specific regulations, in order to reduce the errors caused by failure outside the calibrated area, testing of a higher number of specimens may be considered.

The results presented in the literature indicate that at lower layer thicknesses better interlayer diffusion is achieved, the air voids are smaller, the surface quality is better, and the mechanical properties are higher.

High printing speeds can lead to an inadequate surface quality because of the incomplete solidification of the underlying layers when the top layers are deposited.

Low printing head temperatures can cause incomplete melting, while high printing temperatures can cause unstable material flow from the printing head. Controlling heat flows by using enclosed workspace equipment can reduce the temperature gradients on the build plate.

Upright printed specimens ( $Z Y$ and ZX build orientations) have considerably lower mechanical strength compared to horizontally printed specimens ( $X Y, X Z, Y X$ and $Y Z$ ). The mechanical properties decrease with increases in the specimen positioning angle relative to the build plate.

The use of the alternating raster leads to superior mechanical properties compared to the unidirectional raster. The anisotropic behavior of PLA components made by fused filament fabrication is highly correlated with the raster parameters, build orientation and the type of failure: inter-layer failure, inter-line failure and in-layer/in-line failure.

Author Contributions: Conceptualization and methodology: V.C. and D.F.; data curation and investigation: V.C., D.F., C.-O.M. and G.M.; writing-original draft preparation: V.C.; writing-review and editing: V.C., D.F., C.-O.M. and G.M.; visualization: V.C.; supervision: V.C. and D.F. All authors have read and agreed to the published version of the manuscript.

Funding: This research received no external funding.

Data Availability Statement: Not applicable.

Conflicts of Interest: The authors declare no conflict of interest. 


\section{References}

1. Gordelier, T.J.; Thies, P.R.; Turner, L.; Johanning, L. Optimising the FDM additive manufacturing process to achieve maximum tensile strength: A state-of-the-art review. Rapid Prototyp. J. 2019, 25, 953-971. [CrossRef]

2. International Organization for Standardization. ISO/ASTM 52900:2015; Additive Manufacturing-General PrinciplesTerminology. International Organization for Standardization: Geneva, Switzerland, 2015.

3. Zhang, P.; Wang, Z.; Li, J.; Li, X.; Cheng, L. From materials to devices using fused deposition modeling: A state-of-art review. Nanotechnol. Rev. 2020, 9, 1594-1609. [CrossRef]

4. International Organization for Standardization. ISO 17296-3:2014; Additive Manufacturing-General Principles—Part 3: Main Characteristics and Corresponding Test Methods. International Organization for Standardization: Geneva, Switzerland, 2014.

5. Goh, G.D.; Yap, Y.L.; Tan, H.K.J.; Sing, S.L.; Goh, G.L.; Yeong, W.Y. Process-Structure-Properties in Polymer Additive Manufacturing via Material Extrusion: A Review. Crit. Rev. Solid State Mat. Sci. 2020, 45, 113-133. [CrossRef]

6. Popescu, D.; Zapciu, A.; Amza, C.; Baciu, F.; Marinescu, R. FDM process parameters influence over the mechanical properties of polymer specimens: A review. Polym. Test. 2018, 69, 157-166. [CrossRef]

7. Harris, M.; Potgieter, J.; Archer, R.; Arif, K.M. Effect of Material and Process Specific Factors on the Strength of Printed Parts in Fused Filament Fabrication: A Review of Recent Developments. Materials 2019, 12, 1664. [CrossRef] [PubMed]

8. Vettori, M.; Bergonzi, L. A territorial round-robin experiment for the evaluation of mechanical properties of FDM PLA produced by distributed facilities. In Proceedings of the 49th Italian-Association-for-Stress-Analysis Conference (AIAS), Genova, Italy, 2-5 September 2020; Volume 1038. [CrossRef]

9. Dey, A.; Yodo, N. A Systematic Survey of FDM Process Parameter Optimization and Their Influence on Part Characteristics. J. Manuf. Mater. Process. 2019, 3, 64. [CrossRef]

10. Topcu, I. Mechanical properties of PLA and ABS parts produced with fused filament fabrication method. J. Ceram. Process. Res. 2021, 22, 143-148.

11. Tymrak, B.M.; Kreiger, M.; Pearce, J. Mechanical Properties of Components Fabricated with Open- Source 3-D Printers Under Realistic Environmental Conditions. Mater. Des. 2014, 58, 242-246. [CrossRef]

12. Ebel, E.; Sinnemann, T. Fabrication of FDM 3D objects with ABS and PLA determination of their mechanical properties. Rtejournal 2014, 2014, 1-12. Available online: https://rtejournal.de/paper (accessed on 14 December 2021).

13. Algarni, M.; Ghazali, S. Comparative Study of the Sensitivity of PLA, ABS, PEEK, and PETG's Mechanical Properties to FDM Printing Process Parameters. Crystals 2021, 11, 995. [CrossRef]

14. Kristiawan, R.B.; Imaduddin, F.; Ariawan, D.; Arifin, Z. A review of the fused depostion modeling (FDM) 3D printing: Filament processing, materials, and printing parameters. Open Eng. 2021, 11, 639-649. [CrossRef]

15. Nyiranzeyimana, G.; Mutua, J.M.; Mose, B.R.; Mbuya, T.O. Optimization of process parameters in fused deposition modelling of thermoplastics: A review. Materialwiss. Werkstofftech. 2021, 52, 682-694. [CrossRef]

16. Cuan-Urquizo, E.; Barocio, E.; Tejada-Ortigoza, V.; Pipes, R.B.; Rodriguez, C.A.; Roman-Flores, A. Characterization of the Mechanical Properties of FFF Structures and Materials: A Review on the Experimental, Computational and Theoretical Approaches. Materials 2019, 12, 895. [CrossRef] [PubMed]

17. International Organization for Standardization. ISO/ASTM 52921:2013; Standard Terminology for Additive ManufacturingCoordinate Systems and Test Methodologies. International Organization for Standardization: Geneva, Switzerland, 2013.

18. Hodzic, D.; Pandzic, A.; Hajro, I.; Tasic, P. Strength Comparison of FDM 3D Printed PLA Made by Different Manufacturers. TEM J. 2020, 9, 966-970. [CrossRef]

19. Kuznetsov, V.E.; Solonin, A.N.; Urzhumtsev, O.D.; Schilling, R.; Tavitov, A.G. Strength of PLA Components Fabricated with Fused Deposition Technology Using a Desktop 3D Printer as a Function of Geometrical Parameters of the Process. Polymers 2018, 10, 313. [CrossRef]

20. Wittbrodt, B.; Pearce, J.M. The effects of PLA color on material properties of 3-D printed components. Addit. Manuf. 2015, 8, 110-116. [CrossRef]

21. Spina, R. Performance Analysis of Colored PLA Products with a Fused Filament Fabrication Process. Polymers 2019, 11, 1984. [CrossRef]

22. Torres, J.; Cole, M.; Owji, A.; DeMastry, Z.; Gordon, A.P. An approach for mechanical property optimization of fused deposition modeling with polylactic acid via design of experiments. Rapid Prototyp. J. 2016, 22, 387-404. [CrossRef]

23. Farazin, A.; Mohammadimehr, M. Effect of different parameters on the tensile properties of printed Polylactic acid samples by FDM: Experimental design tested with MDs simulation. Int. J. Adv. Manuf. Technol. 2022, 118, 103-118. [CrossRef]

24. Samykano, M. Mechanical Property and Prediction Model for FDM-3D Printed Polylactic Acid (PLA). Arab. J. Sci. Eng. 2021, 46, 7875-7892. [CrossRef]

25. Pratama, J.; Cahyono, S.I.; Suyitno, S.; Muflikhun, M.A.; Salim, U.A.; Mahardika, M.; Arifvianto, B. A Review on the Reinforcement Methods for Polymeric Materials Processed by Using Fused Filament Fabrication (FFF). Polymers 2021, 13, 4022. [CrossRef] [PubMed]

26. Chacon, J.M.; Caminero, M.A.; Garcia-Plaza, E.; Nunez, P.J. Additive manufacturing of PLA structures using fused deposition modelling: Effect of process parameters on mechanical properties and their optimal selection. Mater. Des. 2017, 124, 143-157. [CrossRef] 
27. Hanon, M.M.; Marczis, R.; Zsidai, L. Influence of the 3D Printing Process Settings on Tensile Strength of PLA and HT-PLA. Period. Polytech-Mech. Eng. 2021, 65, 38-46. [CrossRef]

28. Laureto, J.J.; Pearce, J.M. Anisotropic mechanical property variance between ASTM D638-14 type I and type IV fused filament fabricated specimens. Polym. Test 2018, 68, 294-301. [CrossRef]

29. Khosravani, M.R.; Reinicke, T. Effects of raster layup and printing speed on strength of 3D-printed structural components. In Proceedings of the 1st European-Structural-Integrity-Society (ESIS) Virtual European Conference on Fracture (ECF), Virtual, 29 June-1 July 2020; pp. 720-725. [CrossRef]

30. Kuznetsov, V.E.; Solonin, A.N.; Tavitov, A.; Urzhumtsev, O.; Vakulik, A. Increasing strength of FFF three-dimensional printed parts by influencing on temperature-related parameters of the process. Rapid Prototyp. J. 2020, 26, 107-121. [CrossRef]

31. Lee, C.Y.; Liu, C.Y. The influence of forced-air cooling on a 3D printed PLA part manufactured by fused filament fabrication. Addit. Manuf. 2019, 25, 196-203. [CrossRef]

32. Hasan, M.S.; Ivanov, T.; Vorkapic, M.; Simonovic, A.; Daou, D.; Kovacevic, A.; Milovanovic, A. Impact of Aging Effect and Heat Treatment on the Tensile Properties of PLA (Poly Lactic Acid) Printed Parts. Mater. Plast. 2020, 57, 147-159. [CrossRef]

33. Benwood, C.; Anstey, A.; Andrzejewski, J.; Misra, M.; Mohanty, A.K. Improving the Impact Strength and Heat Resistance of 3D Printed Models: Structure, Property, and Processing Correlationships during Fused Deposition Modeling (FDM) of Poly(Lactic Acid). ACS Omega 2018, 3, 4400-4411. [CrossRef]

34. Behzadnasab, M.; Yousefi, A.A.; Ebrahimibagha, D.; Nasiri, F. Effects of processing conditions on mechanical properties of PLA printed parts. Rapid Prototyp. J. 2020, 26, 381-389. [CrossRef]

35. Liu, Z.G.; Wang, Y.Q.; Wu, B.C.; Cui, C.Z.; Guo, Y.; Yan, C. Critical review of fused deposition modeling 3D printing technology in manufacturing polylactic acid parts. Int. J. Adv. Manuf. Technol. 2019, 102, 2877-2889. [CrossRef]

36. Valerga, A.P.; Batista, M.; Salguero, J.; Girot, F. Influence of PLA Filament Conditions on Characteristics of FDM Parts. Materials 2018, 11, 1322. [CrossRef]

37. Algarni, M. The Influence of Raster Angle and Moisture Content on the Mechanical Properties of PLA Parts Produced by Fused Deposition Modeling. Polymers 2021, 13, 237. [CrossRef]

38. Barrasa, J.O.; Ferrandez-Montero, A.; Ferrari, B.; Pastor, J.Y. Characterisation and Modelling of PLA Filaments and Evolution with Time. Polymers 2021, 13, 2899. [CrossRef]

39. Ambrus, S.; Soporan, R.A.; Kazamer, N.; Pascal, D.T.; Muntean, R.; Dume, A.I.; Marginean, G.M.; Serban, V.A. Characterization and mechanical properties of fused deposited PLA material. Mater. Today-Proc. 2021, 45, 4356-4363. [CrossRef]

40. Pastor-Artigues, M.M.; Roure-Fernandez, F.; Ayneto-Gubert, X.; Bonada-Bo, J.; Perez-Guindal, E.; Buj-Corral, I. Elastic Asymmetry of PLA Material in FDM-Printed Parts: Considerations Concerning Experimental Characterisation for Use in Numerical Simulations. Materials 2020, 13, 15. [CrossRef] [PubMed]

41. ASTM International. ASTM D638-14; Standard Test Method for Tensile Properties of Plastics. ASTM International: West Conshohocken, PA, USA, 2014.

42. International Organization for Standardization. ISO 527-2:2012; Plastics-Determination of Tensile Properties-Part 2: Test Conditions for Molding and Extrusion Plastics. International Organization for Standardization: Geneva, Switzerland, 2012.

43. ASTM International. ASTM D790-10; Standard Test Methods for Flexural Properties of Unreinforced and Rein-forced Plastics and Electrical Insulating Materials. ASTM International: West Conshohocken, PA, USA, 2010.

44. International Organization for Standardization. ISO 178:2019; Plastics-Determination of Flexural Properties. International Organization for Standardization: Geneva, Switzerland, 2019.

45. Sierra, J.; Sanin, D.; Montoya, A.; Villaneda, W. Relation Between Mechanical Properties and 3D Printer Configurations Parameters Using PLA at Open-Source Prusa I3. Int. J. Integr. Eng. 2020, 12, 97-108. [CrossRef]

46. International Organization for Standardization. ISO 527-5:2021; Plastics-Determination of Tensile Properties-Part 5: Test Conditions for Unidirectional Fibre-Reinforced Plastic Composites. International Organization for Standardization: Geneva, Switzerland, 2021.

47. ASTM International. ASTM D3039/D3039M-08; Standard Test Method for Tensile Properties of Polymer Matrix Composite Materials. ASTM International: West Conshohocken, PA, USA, 2008.

48. Valean, C.; Marsavina, L.; Marghitas, M.; Linul, E.; Razavi, J.; Berto, F. Effect of manufacturing parameters on tensile properties of FDM printed specimens. In Proceedings of the 1st Mediterranean Conference on Fracture and Structural Integrity (MedFract), Athens, Greece, 26-28 February 2020; pp. 313-320. [CrossRef]

49. Verbeeten, W.M.H.; Lorenzo-Banuelos, M.; Arribas-Subinas, P.J. Anisotropic rate-dependent mechanical behavior of Poly(Lactic Acid) processed by Material Extrusion Additive Manufacturing. Addit. Manuf. 2020, 31, 100968. [CrossRef]

50. Vidakis, N.; Petousis, M.; Velidakis, E.; Liebscher, M.; Mechtcherine, V.; Tzounis, L. On the Strain Rate Sensitivity of Fused Filament Fabrication (FFF) Processed PLA, ABS, PETG, PA6, and PP Thermoplastic Polymers. Polymers 2020, 12, 2924. [CrossRef]

51. Bayraktar, O.; Uzun, G.; Cakiroglu, R.; Guldas, A. Experimental study on the 3D-printed plastic parts and predicting the mechanical properties using artificial neural networks. Polym. Adv. Technol. 2017, 28, 1044-1051. [CrossRef]

52. Tezel, T.; Kovan, V. Determination of optimum production parameters for 3D printers based on nozzle diameter. Rapid Prototyp. J. 2021, 28, 185-194. [CrossRef]

53. Triyono, J.; Sukanto, H.; Saputra, R.M.; Smaradhana, D.F. The effect of nozzle hole diameter of 3D printing on porosity and tensile strength parts using polylactic acid material. Open Eng. 2020, 10, 762-768. [CrossRef] 
54. Alafaghani, A.; Qattawi, A.; Alrawi, B.; Guzman, A. Experimental Optimization of Fused Deposition Modelling Processing Parameters: A Design-for-Manufacturing Approach. Procedia Manuf. 2017, 10, 791-803. [CrossRef]

55. Alafaghani, A.; Qattawi, A. Investigating the effect of fused deposition modeling processing parameters using Taguchi design of experiment method. J. Manuf. Process. 2018, 36, 164-174. [CrossRef]

56. Altan, M.; Eryildiz, M.; Gumus, B.; Kahraman, Y. Effects of process parameters on the quality of PLA products fabricated by fused deposition modeling (FDM): Surface roughness and tensile strength. Mater. Test. 2018, 60, 471-477. [CrossRef]

57. Bardiya, S.; Jerald, J.; Satheeshkumar, V. The impact of process parameters on the tensile strength, flexural strength and the manufacturing time of fused filament fabricated (FFF) parts. Mater. Today-Proc. 2021, 39, 1362-1366. [CrossRef]

58. Beniak, J.; Krizan, P.; Matus, M. Mechanical properties of biodegradable pla plastic parts produced by 3D printing. MM Sci. J. 2019, 2746-2750. [CrossRef]

59. Cardoso, P.H.M.; Teixeira, B.N.; Calado, V.M.D.; de Oliveira, M.G.; Mendonca, T.D.; Mendonca, R.H.; de Almeida, H.R.O.; Cunha, M.S.; Thire, R. Mechanical and dimensional performance of poly(lactic acid) 3D-printed parts using thin plate spline interpolation. J. Appl. Polym. Sci. 2020, 137, 49171. [CrossRef]

60. Giri, J.; Chiwande, A.; Gupta, Y.; Mahatme, C.; Giri, P. Effect of process parameters on mechanical properties of $3 \mathrm{~d}$ printed samples using FDM process. Mater. Today-Proc. 2021, 47, 5856-5861. [CrossRef]

61. Jaya Christiyan, K.G.; Chandrasekhar, U.; Venkateswarlu, K. Flexural Properties of PLA Components Under Various Test Condition Manufactured by 3D Printer. J. Inst. Eng. (India) Ser. C 2018, 99, 363-376. [CrossRef]

62. Kuznetsov, V.E.; Tavitov, A.G.; Urzhumtsev, O.D.; Mikhalin, M.V.; Moiseev, A.I. Hardware Factors Influencing Strength of Parts Obtained by Fused Filament Fabrication. Polymers 2019, 11, 1870. [CrossRef]

63. Lanzoti, A.; Grasso, M.; Staiano, G.; Martorelli, M. The impact of process parameters on mechanical properties of parts fabricated in PLA with an open-source 3-D printer. Rapid Prototyp. J. 2015, 21, 604-617. [CrossRef]

64. Lokesh, N.; Praveena, B.; Sudheer Reddy, J.; Vikram Kedambadi, V.; Vijaykumar, S. Evaluation on effect of printing process parameter through Taguchi approach on mechanical properties of 3D printed PLA specimens using FDM at constant printing temperature. Mater. Today-Proc. 2021, in press. [CrossRef]

65. Luzanin, O.; Movrin, D.; Stathopoulos, V.; Pandis, P.; Radusin, T.; Guduric, V. Impact of processing parameters on tensile strength, in-process crystallinity and mesostructure in FDM-fabricated PLA specimens. Rapid Prototyp. J. 2019, 25, 1398-1410. [CrossRef]

66. Mazurchevici, S.N.; Pricop, B.; Istrate, B.; Mazurchevici, A.D.; Carlescu, V.; Carausu, C.; Nedelcu, D. Technological Parameters Effects on Mechanical Properties of Biodegradable Materials Using FDM. Mater. Plast. 2020, 57, 215-227. [CrossRef]

67. Milovanovic, A.; Sedmak, A.; Grbovic, A.; Golubovic, Z.; Mladenovic, G.; Colic, K.; Milosevic, M. Comparative analysis of printing parameters effect on mechanical properties of natural PLA and advanced PLA-X material. In Proceedings of the 1st European-Structural-Integrity-Society (ESIS) Virtual European Conference on Fracture (ECF), Virtual, 29 June-1 July 2020; pp. 1963-1968. [CrossRef]

68. Priya, M.S.; Naresh, K.; Jayaganthan, R.; Velmurugan, R. A comparative study between in-house 3D printed and injection molded ABS and PLA polymers for low-frequency applications. Mater. Res. Express 2019, 6, 8.

69. Rajpurohit, S.R.; Dave, H.K. Flexural strength of fused filament fabricated (FFF) PLA parts on an open-source 3D printer. Adv. Manuf. 2018, 6, 430-441. [CrossRef]

70. Rajpurohit, S.R.; Dave, H.K. Analysis of tensile strength of a fused filament fabricated PLA part using an open-source 3D printer. Int. J. Adv. Manuf. Technol. 2019, 101, 1525-1536. [CrossRef]

71. Rajpurohit, S.R.; Dave, H.K. Impact strength of 3D printed PLA using open source FFF-based 3D printer. Prog. Addit. Manuf. 2021, 6, 119-131. [CrossRef]

72. Rodriguez-Panes, A.; Claver, J.; Camacho, A.M. The Influence of Manufacturing Parameters on the Mechanical Behaviour of PLA and ABS Pieces Manufactured by FDM: A Comparative Analysis. Materials 2018, 11, 1333. [CrossRef]

73. Vanaei, H.; Shirinbayan, M.; Deligant, M.; Raissi, K.; Fitoussi, J.; Khelladi, S.; Tcharkhtchi, A. Influence of process parameters on thermal and mechanical properties of polylactic acid fabricated by fused filament fabrication. Polym. Eng. Sci. 2020, 60, $1822-1831$. [CrossRef]

74. Wang, S.H.; Ma, Y.B.; Deng, Z.C.; Zhang, S.; Cai, J.X. Effects of fused deposition modeling process parameters on tensile, dynamic mechanical properties of 3D printed polylactic acid materials. Polym. Test 2020, 86, 106483. [CrossRef]

75. Zisopol, D.G.; Nae, I.; Portoaca, A.I.; Ramadan, I.A. Theoretical and Experimental Research on the Influence of FDM Parameters on Tensile Strength and Hardness of Parts Made of Polylactic Acid. Eng. Technol. Appl. Sci. Res. 2021, 11, 7458-7463. [CrossRef]

76. Yao, T.Y.; Deng, Z.C.; Zhang, K.; Li, S.M. A method to predict the ultimate tensile strength of 3D printing polylactic acid (PLA) materials with different printing orientations. Compos. Pt. B-Eng. 2019, 163, 393-402. [CrossRef]

77. Yao, T.Y.; Ye, J.; Deng, Z.C.; Zhang, K.; Ma, Y.B.; Ouyang, H.J. Tensile failure strength and separation angle of FDM 3D printing PLA material: Experimental and theoretical analyses. Compos. Pt. B-Eng. 2020, 188, 107894. [CrossRef]

78. Yao, T.Y.; Zhang, K.; Deng, Z.C.; Ye, J. A novel generalized stress invariant-based strength model for inter-layer failure of FFF 3D printing PLA material. Mater. Des. 2020, 193, 108799. [CrossRef]

79. Zhao, Y.; Chen, Y.S.; Zhou, Y.J. Novel mechanical models of tensile strength and elastic property of FDM AM PLA materials: Experimental and theoretical analyses. Mater. Des. 2019, 181, 108089. [CrossRef]

80. Ansari, A.A.; Kamil, M. Effect of print speed and extrusion temperature on properties of 3D printed PLA using fused deposition modeling process. Mater. Today-Proc. 2021, 45, 5462-5468. [CrossRef] 
81. Deshwal, S.; Kumar, A.; Chhabra, D. Exercising hybrid statistical tools GA-RSM, GA-ANN and GA-ANFIS to optimize FDM process parameters for tensile strength improvement. CIRP J. Manuf. Sci. Technol. 2020, 31, 189-199. [CrossRef]

82. Durga Prasad Reddy, J.; Mishra, D.; Chetty, N. Strength and Hardness of 3D printed poly lactic acid and carbon fiber poly lactic acid thermoplastics. In Advances in Lightweight Materials and Structures; Kumar, A.P., Dirgantara, T., Krishna, P.V., Eds.; Springer: Singapore, 2020; Volume 8, pp. 625-634. [CrossRef]

83. Hikmat, M.; Rostam, S.; Ahmed, Y.M. Investigation of tensile property-based Taguchi method of PLA parts fabricated by FDM 3D printing technology. Results Eng. 2021, 11, 100264. [CrossRef]

84. Hou, T.; Huang, T.T.; Sun, F.Q.; Wang, S.G. Ultimate tensile strength in fused deposition modeling considering process parameters of flow rate and printing head speed. In Proceedings of the 12th International Conference on Reliability, Maintainability, and Safety (ICRMS), Shanghai, China, 17-19 October 2018; pp. 111-116. [CrossRef]

85. Hsueh, M.H.; Lai, C.J.; Wang, S.H.; Zeng, Y.S.; Hsieh, C.H.; Pan, C.Y.; Huang, W.C. Effect of Printing Parameters on the Thermal and Mechanical Properties of 3D-Printed PLA and PETG Using Fused Deposition Modeling. Polymers 2021, 13, 1758. [CrossRef]

86. Naveed, N. Investigating the Material Properties and Microstructural Changes of Fused Filament Fabricated PLA and Tough-PLA Parts. Polymers 2021, 13, 1487. [CrossRef] [PubMed]

87. Tajarernmuang, T.; Sawangrat, C.; Jomjunyong, S. Effect of process parameters to flexural strength of 3D printed anatomical bone part. In Proceedings of the 10th International Conference on Mechatronics and Manufacturing (ICMM), Bangkok, Thailand, 21-23 January 2019; Chulalongkorn University: Bangkok, Thailand, 2019; Volume 635. [CrossRef]

88. Zakaria, H.; Khan, S.F.; Fee, M.F.C.; Ibrahim, M. Printing temperature, printing speed and raster angle variation effect in fused filament fabrication. In Proceedings of the 6th International Conference on Applications and Design in Mechanical Engineering (ICADME), Penang, Malaysia, 26-27 August 2019; Volume 670. [CrossRef]

89. Zekavat, A.R.; Jansson, A.; Larsson, J.; Pejryd, L. Investigating the effect of fabrication temperature on mechanical properties of fused deposition modeling parts using X-ray computed tomography. Int. J. Adv. Manuf. Technol. 2019, 100, 287-296. [CrossRef]

90. Attolico, M.A.; Casavola, C.; Cazzato, A.; Moramarco, V.; Renna, G. Effect of extrusion temperature on fused filament fabrication parts orthotropic behaviour. Rapid Prototyp. J. 2020, 26, 639-647. [CrossRef]

91. Butt, J.; Bhaskar, R.; Mohaghegh, V. Investigating the effects of extrusion temperatures and material extrusion rates on FFF-printed thermoplastics. Int. J. Adv. Manuf. Technol. 2021, 117, 2679-2699. [CrossRef]

92. Harris, M.; Potgieter, J.; Archer, R.; Arif, K.M. In-process thermal treatment of polylactic acid in fused deposition modelling. Mater. Manuf. Process. 2019, 34, 701-713. [CrossRef]

93. Wach, R.A.; Wolszczak, P.; Adamus-Wlodarczyk, A. Enhancement of Mechanical Properties of FDM-PLA Parts via Thermal Annealing. Macromol. Mater. Eng. 2018, 303, 9. [CrossRef]

94. Liparoti, S.; Sofia, D.; Romano, A.; Marra, F.; Pantani, R. Fused Filament Deposition of PLA: The Role of Interlayer Adhesion in the Mechanical Performances. Polymers 2021, 13, 399. [CrossRef]

95. Vanaei, H.R.; Shirinbayan, M.; Vanaei, S.; Fitoussi, J.; Khelladi, S.; Tcharkhtchi, A. Multi-scale damage analysis and fatigue behavior of PLA manufactured by fused deposition modeling (FDM). Rapid Prototyp. J. 2021, 27, 371-378. [CrossRef]

96. Vanaei, H.R.; Shirinbayan, M.; Deligant, M.; Khelladi, S.; Tcharkhtchi, A. In-Process Monitoring of Temperature Evolution during Fused Filament Fabrication: A Journey from Numerical to Experimental Approaches. Thermo 2021, 1, 332-360. [CrossRef]

97. Bin Ishak, I.; Fleming, D.; Larochelle, P. Multiplane fused deposition modeling: A study of tensile strength. Mech. Based Des. Struct. Mech. 2019, 47, 583-598. [CrossRef]

98. Corapi, D.; Morettini, G.; Pascoletti, G.; Zitelli, C. Characterization of a Polylactic acid (PLA) produced by Fused Deposition Modeling (FDM) technology. Procedia Struct. Integr. 2019, 24, 289-295. [CrossRef]

99. Doungkom, P.; Jiamjiroch, K. Analysis of Printing Pattern and Infiltration Percent over the Tensile Properties of PLA Printed Parts by a Fused Deposition Modelling Printer. In Proceedings of the 9th Thai-Society-of-Mechanical-Engineers International Conference on Mechanical Engineering (TSME-ICoME), Phuket, Thailand, 11-14 December 2018; Volume 501. [CrossRef]

100. Stoica, C.R.; Maier, R.; Istrate, A.; Mandoc, A.C. Assessment of Static Mechanical Properties of Additively Manufactured Polylactic Acid (PLA) on Entry-Level FDM 3D Printer. Mater. Plast. 2021, 58, 176-184. [CrossRef]

101. Dai, S.; Deng, Z.C.; Yu, Y.J.; Zhang, K.; Wang, S.H.; Ye, J. Orthotropic elastic behaviors and yield strength of fused deposition modeling materials: Theory and experiments. Polym. Test 2020, 87, 106520. [CrossRef]

102. Gabor, C.; Pop, M.A.; Magli, D.; Bedo, T.; Munteanu, S.I.; Munteanu, D. The optimization of the production procedure in relation to the mechanical properties of additively manufactured parts. Mater. Today-Proc. 2019, 19, 1008-1013. [CrossRef]

103. Gonabadi, H.; Yadav, A.; Bull, S.J. The effect of processing parameters on the mechanical characteristics of PLA produced by a 3D FFF printer. Int. J. Adv. Manuf. Technol. 2020, 111, 695-709. [CrossRef]

104. Hsueh, M.H.; Lai, C.J.; Chung, C.F.; Wang, S.H.; Huang, W.C.; Pan, C.Y.; Zeng, Y.S.; Hsieh, C.H. Effect of Printing Parameters on the Tensile Properties of 3D-Printed Polylactic Acid (PLA) Based on Fused Deposition Modeling. Polymers 2021, $13,2387$. [CrossRef]

105. Song, Y.; Li, Y.; Song, W.; Yee, K.; Lee, K.Y.; Tagarielli, V.L. Measurements of the mechanical response of unidirectional 3D-printed PLA. Mater. Des. 2017, 123, 154-164. [CrossRef]

106. Tronvoll, S.A.; Welo, T.; Elverum, C.W. The effects of voids on structural properties of fused deposition modelled parts: A probabilistic approach. Int. J. Adv. Manuf. Technol. 2018, 97, 3607-3618. [CrossRef] 
107. Kiendl, J.; Gao, C. Controlling toughness and strength of FDM 3D-printed PLA components through the raster layup. Compos. Pt. B-Eng. 2020, 180, 107562. [CrossRef]

108. Naveed, N. Investigate the effects of process parameters on material properties and microstructural changes of 3D-printed specimens using fused deposition modelling (FDM). Mater. Technol. 2021, 36, 317-330. [CrossRef]

109. Ayatollahi, M.R.; Nabavi-Kivi, A.; Bahrami, B.; Yahya, M.Y.; Khosravani, M.R. The influence of in-plane raster angle on tensile and fracture strengths of 3D-printed PLA specimens. Eng. Fract. Mech. 2020, 237, 106905. [CrossRef]

110. Cakan, B.G. Effects of raster angle on tensile and surface roughness properties of various FDM filaments. J. Mech. Sci. Technol. 2021, 35, 3347-3353. [CrossRef]

111. Andrzejewska, A.; Pejkowski, L.; Topolinski, T. Tensile and Fatigue Behavior of Additive Manufactured Polylactide. 3D Print. Addit. Manuf. 2019, 6, 272-280.

112. Fayazbakhsh, K.; Movahedi, M.; Kalman, J. The impact of defects on tensile properties of 3D printed parts manufactured by fused filament fabrication. Mater. Today Commun. 2019, 18, 140-148. [CrossRef]

113. Mohan, R.G.; Santhosh, K.; Iyer, R.V.; John, L.K.; Ramu, M. Comparitive analysis of mechanical properties of FDM printed parts based on raster angles. Mater. Today-Proc. 2021, 47, 4730-4734. [CrossRef]

114. Oviedo, A.M.; Puente, A.H.; Bernal, C.; Perez, E. Mechanical evaluation of polymeric filaments and their corresponding 3D printed samples. Polym. Test. 2020, 88, 106561. [CrossRef] 